\title{
Viscoplastic dam breaks and the Bostwick consistometer
}

\author{
N. J. Balmforth, ${ }^{\text {a }}$ R. V. Craster, ${ }^{b}$ P. Perona, ${ }^{c}$ A. C. Rust ${ }^{d}$ \\ \& R. Sassi ${ }^{\mathrm{e}}$

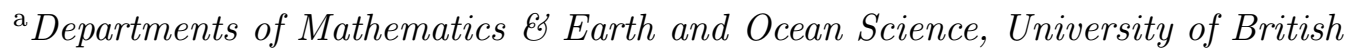 \\ Columbia, Vancouver, Canada \\ ${ }^{\mathrm{b}}$ Department of Mathematics, Imperial College London SW7 2AZ, UK \\ ${ }^{\mathrm{c}}$ Institute of Hydromechanics and Water Resources Management (IHW), ETH \\ Zurich, Switzerland \\ ${ }^{\mathrm{d}}$ Department of Earth and Ocean Science, University of British Columbia, \\ Vancouver, Canada \\ e Dipartimento di Tecnologie dell'Informazione, Università di Milano, Crema, Italy
}

\begin{abstract}
We present a theoretical and experimental analysis of the dam break of a viscoplastic fluid in a horizontal channel. A shallow, slow fluid model based on the Herschel-Bulkley constitutive law allows one to characterize the early and late stages of the flow, the final state and the dependence on yield stress and nonlinear viscosity. A particular diagnostic is advanced (time ratios based on the length of time required for the fluid to slump certain distances from the broken dam) that may assist an experimentalist to unravel those dependences. Experiments are conducted with cornsyrup, and aqueous suspensions of xanthan gum, kaolin, carbopol, cornstarch and apple puree. Cornsyrup xanthan gum and kaolin show fair quantitative agreement with theory. Carbopol compares less favourably, due primarily to inertial effects which are missing from the theory. The results for cornstarch confirm that it is shear thickening, but its detailed rheology remains unknown (and unexplored). Apple puree also appears to compare well with theory, although repeating the dam break in a roughened channel leads to substantially different results, suggesting that fluid separation can induce effective wall slip (a problem that also probably plagues the Bostwick device). Finally, theory is compared with Bostwick tests with fruit puree, with limited success.
\end{abstract}




\section{Introduction}

The breaking of a dam to release a reservoir of viscous fluid in a channel is a classical problem in shallow-water fluid mechanics owing to its mathematical tractability and numerous applications in hydraulic engineering and elsewhere. Although much less attention has been focused on the corresponding non-Newtonian version, a number of industrial and geophysical problems surround the slump of a viscoplastic fluid within a channel, including certain emplacements of concrete and lava, and the flow of a muddy river.

In food science, the problem has actually been reformulated as a rheometric device, the so-called "Bostwick test", which is widely used by government agencies to define technically common terms such as ketchup (see, for example, Perona [17] and the references therein). Here, one releases the fluid in a specially manufactured channel (the "Bostwick consistometer") and one then measures the distance the fluid has slumped after a certain time interval, usually 30 seconds; this gives the "Bostwick consistency", $B_{30}$ (tomato puree, for example, can only be considered as a "ketchup" provided that it has a $B_{30}$ measurement of $14 \mathrm{~cm}$ or less, at $20^{\circ} \mathrm{C}$, according to the U. S. Food and Drug Administration). Unfortunately, as it is based on a single measurement, it is impossible to unravel detailed information about the fluid rheology from this measurement. In particular, one cannot distinguish a fluid yield stress from the effects of a rate-dependent viscosity. Moreover, little theoretical analysis has been presented to compare with experiments in this or related devices. The purpose of the present article is to venture some way in this particular direction.

More specifically, we combine a theoretical exploration of dam breaks of viscoplastic fluid in a channel with some simple experiments using prototypical viscoplastic materials, and with actual Bostwick tests. The theory is based on a shallow, slow approximation to the governing equations (e.g. $[2,3,13])$ which offers a compact description to explore the slump of a viscoplastic fluid described by the Herschel-Bulkley constitutive law. In the shallow, slow approximation, the fluid flow is controlled by the vertical shear stress and the side walls of the channel play no role; thus, the dam break becomes equivalent to the release of a two-dimensional sheet of fluid.

The experiments are designed to compare more closely with the theory than the Bostwick test: the channel is relatively wide and the fluid relatively shallow compared to the usual Bostwick set-up, bringing the configuration closer to the conditions required by the theoretical formulation. At the end of the day, we have some success in connecting theory with this experiment, although there are some significant discrepancies associated with inertia, side-wall drag and wall slip on the channel's floor. The Bostwick tests themselves are described 
more fully by Perona [17], the focus being on the slumps of various kinds of fruit purees and comparisons with a dimensional analysis of the problem. Here we attempt a more detailed comparison with theory.

\section{Mathematical formulation}

Consider the slow flow over a horizontal surface of a planar sheet of incompressible, non-Newtonian fluid with a rheology given by the Herschel-Bulkley constitutive law. We orientate a $(x, z)$-coordinate system with $z$ pointing up and the lower surface occupying the $z=0$ plane; $(u, w)$ denote the components of the fluid velocity field. The fluid density is $\rho$, and $g$ is the gravitational acceleration.

When the fluid layer is relatively shallow, the stresses are dominated by the vertical shear stress, $\tau_{x z}$, and the deformation rates by $u_{z} \equiv \partial u / \partial z$, in which case, the Herschel-Bulkley law reduces to

$$
\tau_{x z}=\eta\left(u_{z}\right) u_{z}+\tau_{y} \operatorname{sgn}\left(u_{z}\right), \quad \text { if }\left|\tau_{x z}\right| \geq \tau_{y}
$$

and $u_{z}=0$ if $\left|\tau_{x z}\right|<\tau_{y}$, where $\tau_{y}$ is the yield stress, $\eta\left(u_{z}\right)=K\left|u_{z}\right|^{n-1}$ is the nonlinear viscosity, $K$ the consistency and $n$ a power-law index that gauges the degree of shear thinning or thickening. If, moreover, the flow is sufficiently slow to neglect inertia, then the full governing equations of the fluid can be reduced to a single evolution equation for the fluid depth, $h(x, t)$ :

$$
h_{t}=\left(\frac{\rho g}{K}\right)^{\frac{1}{n}} \frac{\partial}{\partial x}\left[\frac{n\left|h_{x}\right|^{1 / n-1} Y^{1+1 / n}}{(n+1)(2 n+1)}[(1+2 n) h-n Y] h_{x}\right] \text {, }
$$

where

$$
Y=h-\frac{\tau_{y}}{\rho g\left|h_{x}\right|}
$$

is somewhat like a yield surface $^{1}$, and the subscripts on $h$ denote partial derivatives (e.g. $[2,5,13])$.

$\overline{1 \text { Below }} z=Y(x, t)$, the fluid is fully yielded and shears, whereas for $z>Y(x, t)$, the fluid is nearly unyielded and stresses lie close to the yield stress. Because the fluid above $z=Y$ is not truly rigid, this region is a "pseudo-plug" and $z=Y$ is not a real yield surface, but a "fake" one [2]. 
We solve (2) subject to no-flux conditions at $x=0$ (the back wall), $h \rightarrow 0$ at the fluid front, and with an initial condition in the form of a step (i.e. a dam-break problem) with depth $H$ and length $L$ :

$$
h=\left\{\begin{array}{l}
H, 0 \leq x \leq L \\
0, \quad x>L
\end{array}\right.
$$

It is important to appreciate the implications of the thin-layer scaling that leads to (2): all extensional stress and horizontal shear stresses are neglected in arriving at this result. Consequently, the model breaks down when the fluid layer becomes too steep, or when there is significant horizontal shear. Dam breaks with large yield stress create the setting for steep slumps, whereas horizontal shear necessarily plays a role close to the sides of the channel to ensure that a no-slip boundary condition is maintained. Because the additional resistance stemming from the sidewalls is ignored, the thin-layer model predicts that the fluid remains planar in the dam break (given that it is initially so), even had the theory been cast in fully three-dimensional form $[2,3]$.

\subsection{Dimensionless formulation}

By defining dimensionless variables as:

$$
x=L \hat{x}, \quad z=H \hat{z}, \quad h=H \hat{h}, \quad Y=H \hat{Y}, \quad t=\frac{L}{H}\left(\frac{K L}{\rho g H^{2}}\right)^{\frac{1}{n}} \hat{t},
$$

we rewrite the evolution equation in a standard form with only two parameters ( $n$ and a dimensionless yield stress parameter). On dropping the hat decoration, the non-dimensional version of (2) becomes:

$$
h_{t}=\frac{\partial}{\partial x}\left[\frac{n\left|h_{x}\right|^{1 / n-1} Y^{1+1 / n}}{(n+1)(2 n+1)}[(1+2 n) h-n Y] h_{x}\right], \quad Y=h-\frac{B}{\left|h_{x}\right|},
$$

where $B$ is a scaled yield stress or Bingham number,

$$
B=\frac{\tau_{y} L}{\rho g H^{2}}
$$

(given the choice of units for speed, this dimensionless group can be recognized as the ratio of yield stress to viscous stress, the customary definition of Bingham number), and the initial condition becomes $h(x, 0)=1$ for $0<x<1$ (and zero beyond). 


\section{Theoretical slumps}

\subsection{Sample initial-value problems}

We solve the evolution equation numerically using a finite-difference scheme which is a variant of that described in the appendix of [4]; we add some brief notes on the scheme in Appendix B. Before proceeding with further analysis, we whet the reader's appetite by first displaying some sample results. As could probably be anticipated, the slumps fall into two classes: when the yield stress is not so large, the fluid flows a relatively long distance and the whole mass participates in the slump. Indeed, a Newtonian fluid spreads indefinitely unless one includes the joint effects of surface tension and imperfect wetting of the substrate (a formulation of the yield-stress problem that incorporates surface tension is presented by [6]). On the other hand, when the yield stress is large enough, it is capable of bringing the fluid to rest before the material at the rear end of the channel has even slumped. Thus, "partial" slumps occur in which an unyielded region adjacent to the back wall remains static throughout.

The two scenarios are illustrated in figure 1, which shows snapshots of the fluid depth, $h$, and fake yield surface, $Y$, for two values of Bingham number $(0.1$ and 1), with $n=1$. In both examples, the fluid slumps to a final stationary profile for which $Y \rightarrow 0$ and $h \rightarrow h_{\infty}(x)$, where

$$
h_{\infty}(x)=\sqrt{(3 B)^{2 / 3}-2 B x} \quad \text { if } B<\frac{1}{3}
$$

(i.e. a full slump), or

$$
h_{\infty}(x)=\left\{\begin{array}{ll}
\sqrt{(6 B+1-6 B x) / 3}, & 1-(3 B)^{-1} \leq x \leq 1+(6 B)^{-1} \\
1, & 0<x<1-(3 B)^{-1},
\end{array} \quad \text { if } B \geq \frac{1}{3}(9)\right.
$$

(a partial slump). Note that this profile relies purely on the yield-stress part of the constitutive law and is independent of $n$. Indeed, it is valid for any rate-dependent viscosity. 

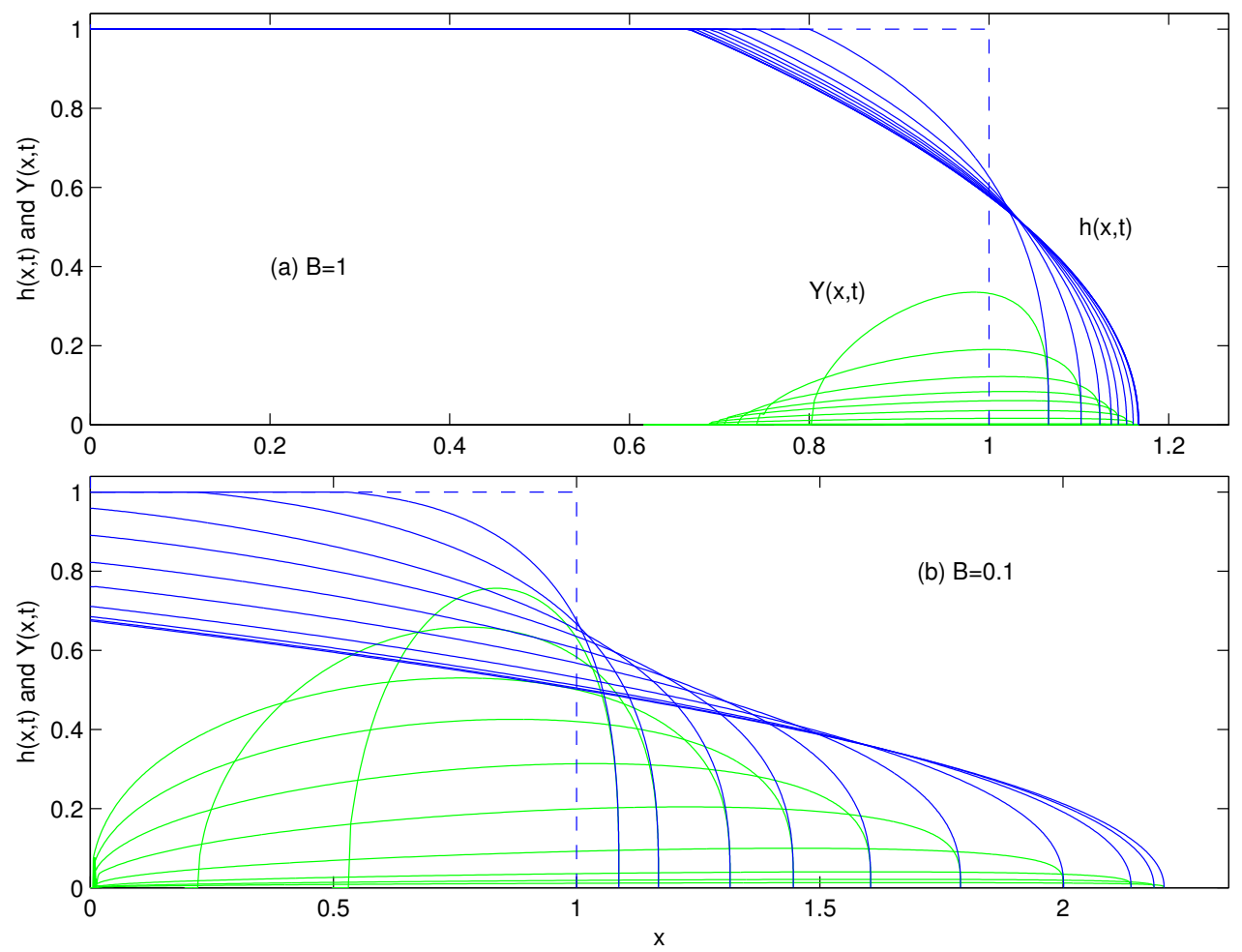

Fig. 1. Snapshots of depth, $h(x, t)$, and fake yield surface, $Y(x, t)$, for (a) $B=1$ and (b) $B=0.1$, with $n=1$. In (a), the time instants are $t=0.1,0.4,0.9,1.6,2.5$, 5, 10, 100, 260, 500 and 830. In (b), the times are $t=0.1,0.4,1.6,3.6,8,60,200$, 420 and 720 . The dashed lines show the initial step.

\subsection{The viscous picture}

When the yield stress is set to zero $(B=0)$, the evolution equation reduces to

$$
h_{t}=\frac{\partial}{\partial x}\left[\frac{n\left|h_{x}\right|^{1 / n-1} h^{2+1 / n}}{(2 n+1)} h_{x}\right]
$$

giving the thin-layer model for a power-law fluid. For $n=1$, we recover a well-known equation for Newtonian fluids [12]; the power-law problem was considered previously by McKarthy and Seymour [16] and Debiane \& Piau [18].

There are two similarity solutions to equation (10) that characterize the short and long-time behaviour of a typical slump. Both take the form,

$$
h=\frac{1}{t^{\alpha}} f(\varpi), \quad \varpi=\frac{x-1}{t^{\beta}} .
$$


The powers, $\alpha$ and $\beta$ are fixed by the requirement that the similarity form succeeds as a solution to the partial differential equation, which demands that $n=\alpha(n+2)+\beta(n+1)$, and a global constraint such as conservation of mass,

$$
M:=\int_{0}^{\infty} h(x, t) d x \rightarrow t^{\beta-\alpha} \int_{0}^{\infty} f(\varpi) d \varpi
$$

Thus, one expects that $\alpha=\beta$ for a constant volume release, which then gives $\alpha=\beta=n /(2 n+3)(c f[12])$. This is the long-time similarity solution, from which we can further extract the explicit form,

$$
h=t^{-n /(2 n+3)}\left(\frac{2 n+1}{2 n+3}\right)^{\frac{n}{2+n}}\left(\frac{n+2}{n+1}\right)^{\frac{1}{2+n}}\left[\Upsilon^{n+1}-\frac{(x-1)^{n+1}}{t^{n(n+1) /(2 n+3)}}\right]^{\frac{1}{2+n}}
$$

where $\varpi=\Upsilon$ denotes the fluid edge,

$$
\Upsilon^{2 n+3}=\frac{(n+1)^{n+3}(2 n+3)^{n}}{(n+2)(2 n+1)^{n}}\left[\beta\left(\frac{1}{n+1}, \frac{n+3}{n+2}\right)\right]^{-n-2},
$$

and $\beta(r, s)$ denotes the beta-function. For example, if $n=1, \Upsilon \approx 1.1329$, giving, in real space, the edge, $X \sim 1+1.1329 t^{1 / 5}$.

The short-time similarity solution arises from a different choice for $\alpha$ and $\beta$ : over early times, the fluid feels little effect from the backwall, and the initial condition resembles an infinitely wide step. In this circumstance, one cannot apply mass conservation to constrain $\alpha$ and $\beta$. However,

$$
1=[h(x, t)]_{x \rightarrow-\infty}=t^{-\alpha}[f(\varpi)]_{\varpi \rightarrow-\infty}
$$

which demands that $\alpha=0$, and so $\beta=n /(n+1)$. One cannot find $f(\varpi)$ in closed form in this case. The solution decays like $(1-\varpi / \Upsilon)^{1 /(n+2)}$ near the front edge. For $n=1$, numerical results indicate that $\Upsilon \approx 0.2845$, and so the advancing fluid edge is approximately given by $X \sim 1+0.2845 t^{1 / 2}$, over early times. At the back of the slump, we estimate that

$$
f \sim 1-F, \quad F_{\varpi} \sim\left[(n-1) \varpi^{2}\right]^{n /(1-n)},
$$

which implies an algebraically decaying "tail" for shear thickening fluid $(n>$ $1)$, an exponential one for the Newtonian case $(n=1)$, and a finite backpropagating edge for shear thinning fluid $(n<1)$. This behaviour explains why the solution barely feels the backwall, and is reproduced in the numerical computations. 
One should bear in mind that the short-time similarity solution may fail at the very earliest times where inertia is important. If inertia dominates at such times, one expects an initial linear-in-time scaling for the fluid edge, based on the shallow-water speed scale, $\sqrt{g H}[14]$.

\subsection{Effects of yield stress and nonlinear viscosity}

The introduction of yield stress disturbs the simple picture offered by the viscous similarity solutions. All the slumps begin initially with a phase adequately described by the viscous theory because surface slopes are so large near the initial step. However, thereafter, the yield stress becomes increasingly important and ultimately triggers a transition to a state dominated by the yield stress in which the fluid converges to the final state, $h=h_{\infty}(x)$. Unfortunately, numerical computations of the initial-value problem are unable to say with certainty exactly how the fluid reaches its final state because the computation is invariably controlled by the small numerical diffusion that is implicit in the computational algorithm. Avoiding this would require an implementation of an augmented Lagrangian scheme, or the like, which we have not done here. Despite this, the numerical computations do suggest the long-time dependence, $\left(h-h_{\infty}, Y\right) \sim t^{-1}$, as the slump converges to its final state. This finding can be backed up with a perturbation analysis with gives a clear indication of how the slump eventually comes to rest (see Matson \& Hogg [15] and the summarizing details in appendix A).

We characterize the transition to the yield-stress dominated state in further detail by tracing the fluid edge, $x=X(t)$, and maximum depth, $h(0, t)$. Sample results are shown in figure 2 for $n=1$. We estimate the "slump time" by determining when the fluid edge reaches a fixed, small fraction of its final value, $X_{\infty}=1+(6 B)^{-1}$ for $B>1 / 3$ or $X_{\infty}=(9 / 8 B)^{1 / 3}$ for $B<1 / 3$. For example, if $X\left(T_{\text {stop }}\right)=1+0.99\left(X_{\infty}-1\right)$, then $t=T_{\text {stop }}$ measures the time taken for the runout, $X-1$, to reach 99 percent of its final value. This quantity is plotted against $B$ in the first panel of figure 3 .

The data in figure 3 suggests that the stopping time roughly follows two different power laws depending on whether the entire fluid layer participated in the slump or not. For partial slumps, the data closely suggests a scaling $T_{\text {stop }} \sim B^{-2}$. The complete slumps, on the other hand plausibly converge to the alternative scaling, $T_{\text {stop }} \sim B^{-5 / 3}$, as $B$ becomes very small. These scalings can be rationalized as follows ( $c f$. Lyman, Kerr \& Griffiths [14]): Yield stresses become important through $Y$ when $B \sim-h h_{x}$. If we use the viscous similarity solutions to evaluate $h h_{x}$, then we arrive at the scalings $B \sim t^{1 / 2}$ for the early time similarity solution, and $B \sim t^{3 / 5}$ for the later-time one. In other words, the stopping-time scaling reflects when one expects yield stresses to break the 

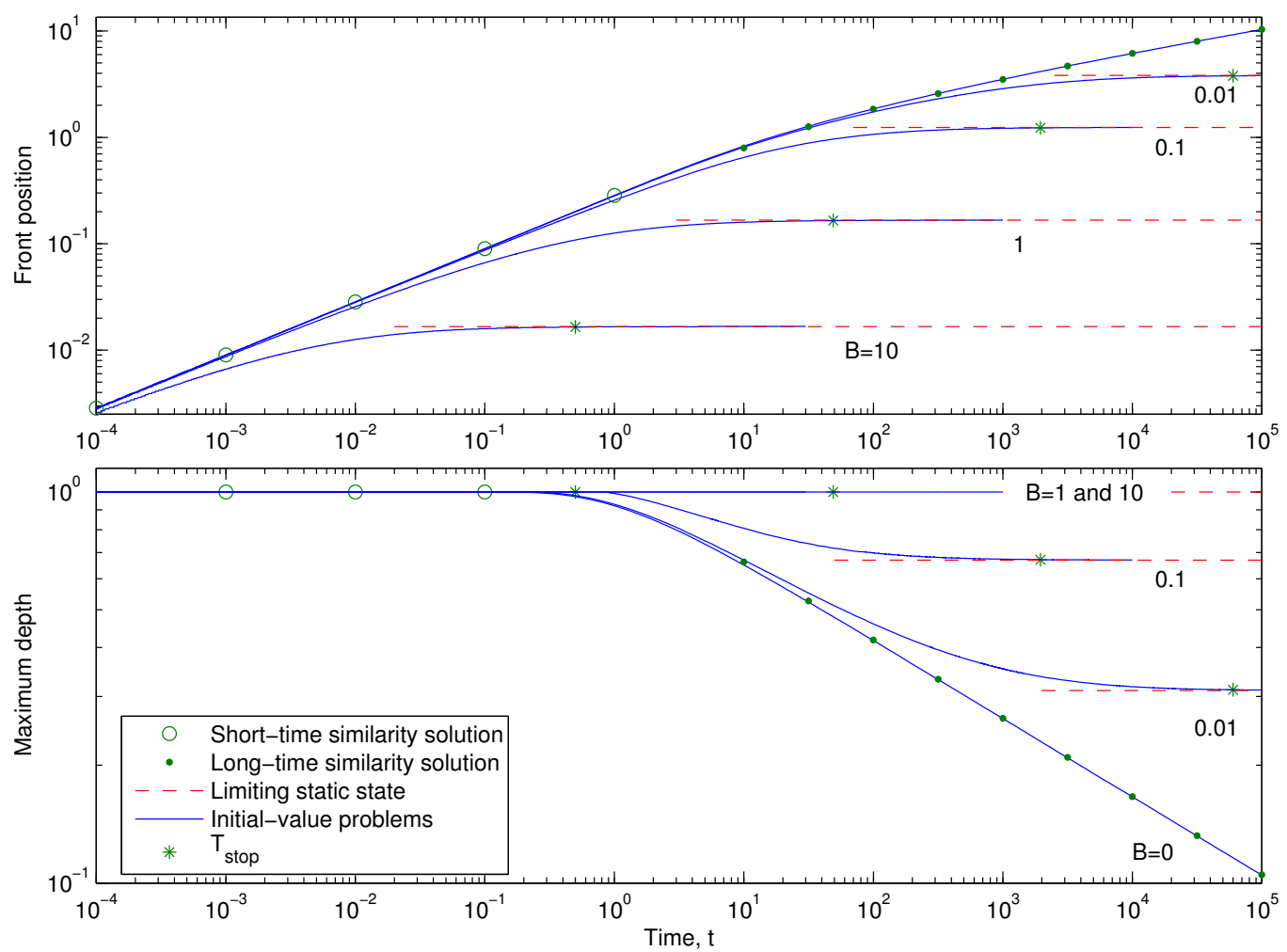

Fig. 2. Evolution of (a) the fluid edge, $X-1$, and (b) maximum depth, $h(0, t)$, for slumps with $B=0,0.01,0.1,1$ and 10 . The horizontal dashed lines show the expected final values, and the stars the values for $t=T_{\text {stop }}$, a convenient marker for when the slump has largely stopped (given by $X(t)=1+0.99\left(X_{\infty}-1\right)$ ). The circles and dots show the short and long-time similarity solutions for viscous fluid.

similarity solution. A more quantitative prediction for the stopping time can be extracted by considering in detail the convergence to the final state via Matson \& Hogg's [15] perturbation theory. A summary of the relevant results of this analysis are presented in Appendix A, and the theoretical predictions are included in figure 3 .

The second panel of figure 3 presents data more in line with usual practice with the Bostwick rheometer: the progression of the slump after a fixed time interval is monitored. Normally, this measurement is performed in real time, rather than with the dimensionless time of the computations. But if we adopt the same philosophy, then we may monitor the slump length after a given (dimensionless) time interval to arrive at an alternative characterization of the slump. Slump lengths after time intervals of 10, 20, 100 and 1000 units, are shown in the second panel as a function of $B$. Should the slump have converged to its final state during the set time interval, then the slump length is simply given by its final value $\left(X_{\infty}(B)=1+(6 B)^{-1}\right.$ or $(9 / 8 B)^{1 / 3}$, depending on whether $B$ exceeds $1 / 3$ or not, respectively). These curves are also included in the figure. As $B$ decreases, the slump length switches from its static value towards the Newtonian one (indicated by the horizontal dashed lines in the 

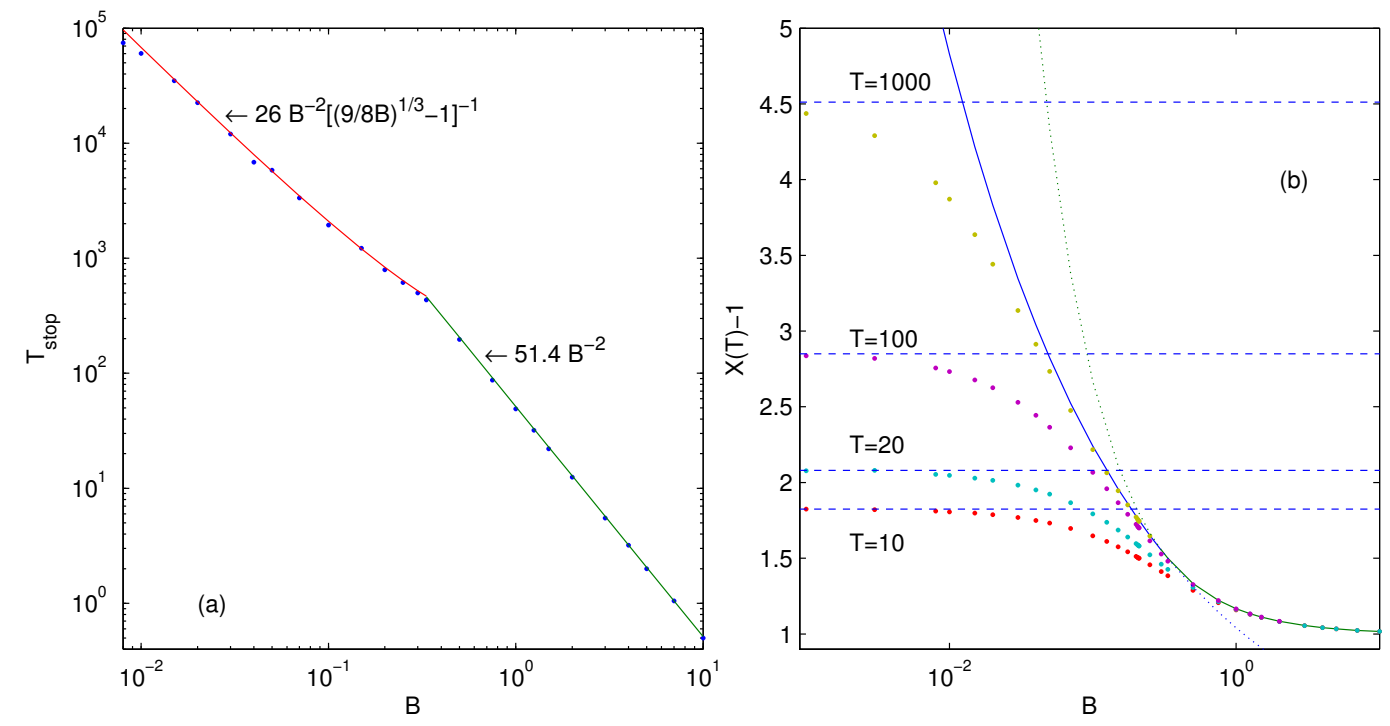

Fig. 3. Data from computations of a suite of initial-value problems showing (a) "stopping time", $T_{\text {stop }}$, and (b) runout length, $X(t)-1$, over fixed time intervals, against Bingham number $B$, for $n=1$. For (a), the lines show the dependences expected from the perturbation theory outlined in Appendix A. In (b), if the slump has converged largely to its final state over the fixed time interval, then the runout approximately measures the final value, which is $(6 B)^{-1}$ or $(9 / 8 B)^{1 / 3}-1$ depending on whether $B>1 / 3$ or $B<1 / 3$, respectively. These curves are shown by the solid lines (the dotted lines show the continuations of each curve outside the range of validity). The horizontal lines indicate the Newtonian runout lengths.

figure).

All preceding results refer to the Bingham case, $n=1$. When we allow also for shear thinning or thickening, there can be a significant influence on the progression of the dam break. In particular, the dramatic increase of the viscosity as a shear-thinning $(n<1)$ fluid brakes to rest significantly prolongs the duration of the slump. Conversely, when $n>1$ and the fluid shear thickens, the relatively slow viscosity in the approach to the final state promotes the importance of the yield stress and halts the slump at earlier times. Both trends are illustrated by the solutions presented in figure 4 .

\subsection{Back to real space}

Given the data of figure 3, we may restore dimensions to find the real-space run-out distance given a particular (dimensional) time interval. In principle, one could then try to use this information to extract details of the viscosity and yield stress given actual measurements. Clearly, because one is attempting to deduce multiple parameter values from a single measurement, this extraction must be problematic. Nevertheless, when the run-out is close to its final 

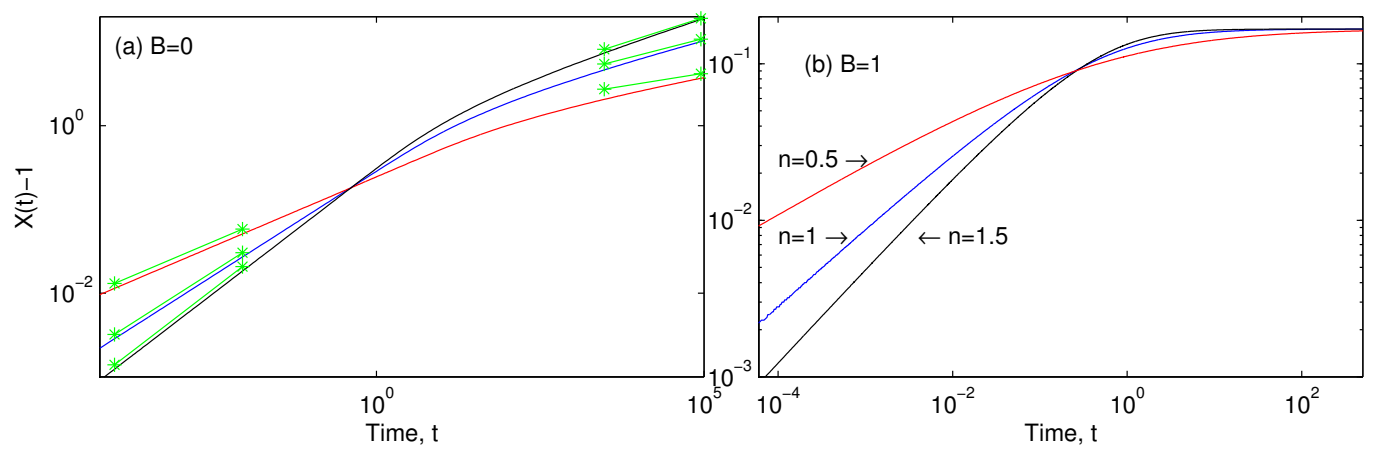

Fig. 4. Run-out distances from the gate, $X(t)-1$, against time for three values of $n$ (as indicated), with (a) $B=0$ and (b) $B=1$. In (a), the similarity scalings are also indicated.

dimensional value, $x_{\infty}$, then we may read off the value of $B$, and hence $\tau_{y}$, from $x_{\infty} / L=X_{\infty}=1+(6 B)^{-1}$ if $X_{\infty}<3 / 2$, or $(9 / 8 B)^{1 / 3}$ if $X_{\infty}>3 / 2$. The precise time interval over which the experiment is conducted is then irrelevant, provided it is sufficiently long that the slump has reached its final state.

If the slump has not converged to the final state, then the procedure is inherently ambiguous as can be seen from figure 4: This picture is presented in dimensionless time, but restoring the dimensions merely scales the axes. It would be straightforward to scale the curves for different $n$ with different values of the consistency, $K$, so that they all crossed at a particular dimensional distance and time (indeed, even in dimensionless variables, the curves already cross close to a single point). Thus, it would be impossible to tell apart shear thinning or thickening fluids, even if they had the same yield stress (as in the figure).

This raises the question of whether multiple measurements can be used to extract more information than just the yield stress. From a theoretical perspective, the simplest route to such a protocol is to measure the progression of the front, recording the time taken to pass various stations downstream of the gate. In dimensionless variables, the time taken to cross a station a distance, $S$, from the gate is given by $T(S ; B)$. Restoring the dimensions, we have a real time,

$$
t(s)=\frac{L}{H}\left(\frac{K L}{\rho g H^{2}}\right)^{\frac{1}{n}} T(s L ; n, B),
$$

denoting the real-space distance travelled as $s=S / L$. Given two such measurements at stations of distances, $s_{j}$ and $s_{k}$, from the gate, we may record the time ratio,

$$
\frac{t\left(s_{j}\right)}{t\left(s_{k}\right)}=\frac{T\left(s_{j} L ; n, B\right)}{T\left(s_{k} L ; n, B\right)},
$$

which removes the consistency $K$ from the problem, leaving one with the need to determine only $n$ and $B$. 


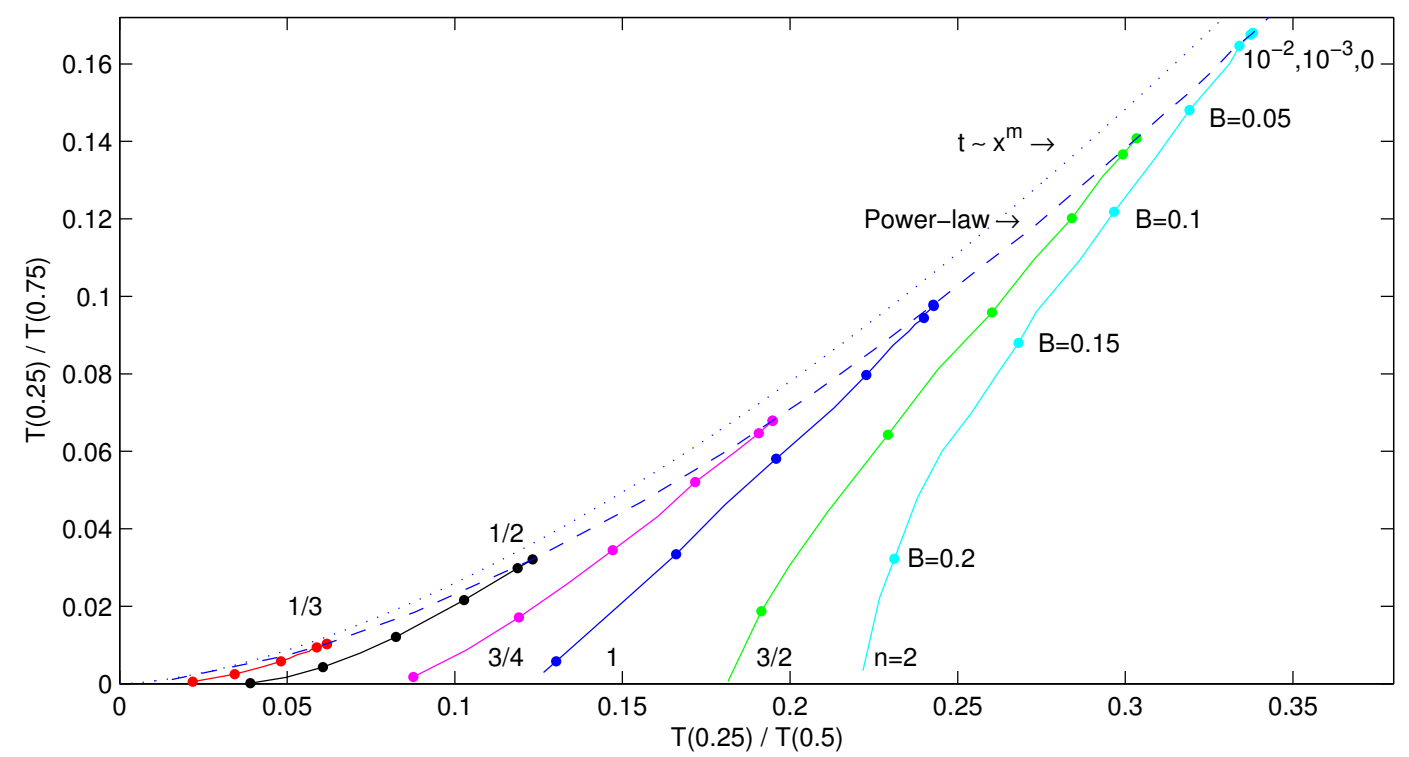

Fig. 5. The time ratios $t\left(s_{1}\right) / t\left(s_{2}\right) \equiv T(0.25) / T(0.5)$ and $t\left(s_{1}\right) / t\left(s_{3}\right) \equiv T(0.25) / T(0.75)$, plotted against one another for suites of initial-value problems with varying $B$ and $n$. Each curve is a different $n$ (as labelled); the points along each curve indicate the $B$-values of $0.2,0.15,0.1,0.05,0.01,10^{-3}$ and 0 . The dashed line shows results for power-law fluid $(B=0)$, and the dotted line indicates the results expected if there was a simple algebraic dependence, $t \sim x^{m}$, for varying $m$.

It turns out that an interesting diagnostic plot for those parameters is afforded by choosing three stations, distances $s_{j}=j L / 4$ from the gate, $j=1,2$ and 3 , and measuring the two ratios, $t_{1} / t_{2} \equiv t\left(s_{1}\right) / t\left(s_{2}\right) \equiv T(0.25) / T(0.5)$ and $t_{1} / t_{3} \equiv t\left(s_{1}\right) / t\left(s_{3}\right) \equiv T(0.25) / T(0.75)$. These time ratios can then be plotted against one another; a plot of this kind is shown in figure 5. The data all fall into a curved wedge on the $\left[t\left(s_{1}\right) / t\left(s_{2}\right), t\left(s_{1}\right) / t\left(s_{3}\right)\right]$ plane, with yield stress monotonically decreasing both ratios. The more shear thinning cases lie nearer the apex at the origin, and the slumps of the thickening fluids give the larger ratios. In principle, the organization of this plot could be used to identify $n$ and $B$. Given those, the actual recorded times imply $K$. Thus, one can use the plot to diagnose the parameters of a Herschel-Bulkley fit to experimental data. Below, we give a brief evaluation of whether this is feasible.

\section{Experiments}

To complement the theory, we conducted some exploratory experiments in which we released a reservoir of fluid in a horizontal channel (see figure 6). We 
used five fluids: cornsyrup and aqueous suspensions of xanthan gum, kaolin, ${ }^{2}$ carbopol, and cornstarch. The channel was $10 \mathrm{~cm}$ wide and $6 \mathrm{~cm}$ deep (although we also performed a limited number of dam breaks in a channel with half the width to judge the effect of side-wall resistance, as reported below). Cornsyrup is a Newtonian control fluid, xanthan gum is a standard shearthinning material, and kaolin and carbopol are proto-typical yield-stress fluids. We also used cornstarch as an example of a fluid that appears to be shear thickening (although its rheology and flow behaviour are actually rather more complex [1]). In addition to these standard fluids, we also used a fruit puree (slightly diluted apple sauce), primarily to establish a firmer connection with Perona's Bostwick tests [17]; we delay discussion of these results until the next section.

For each experiment, the dam was created by holding an obstruction (made of cardboard and waterproofed by covering it with plastic tape) in the channel and then removing it vertically as quickly as possible at time " $t=0$ ". We repeated each experiment a number of times to check that variations in the way that the dam was withdrawn did not appreciably affect the behaviour. Based on the results, we are confident that the details of the dam release are not significant, especially compared to uncertainties in the detailed rheology of the materials used. Admittedly, our protocol is on the crude side, involving little more than tape, cardboard, the channel and a video camera. However, our purpose was in part to judge how far one could proceed with such a rudimenary experimental setup, in the interest of assessing its robustness.

\subsection{Cornsyrup}

We used two varieties of cornsyrup with quite different viscosities (of order $1 \mathrm{~Pa}$ sec and $100 \mathrm{~Pa}$ secs at room temperature). This material slumps slowly down the channel when released, and the progression of its front is well fit by the short-time similarity solution, $x \sim t^{1 / 2}$ (see figure 7 ). The time ratios, $t_{1} / t_{2} \equiv$ $t\left(s_{1}\right) / t\left(s_{2}\right)$ and $t_{1} / t_{3} \equiv t\left(s_{1}\right) / t\left(s_{3}\right)$, are displayed in figure 8 and are close to $1 / 4$ and $1 / 9$, the values expected from the short-time similarity solution and as predicted by the initial-value computations. The more viscous cornsyrup, which has lower values for the time ratios than the thinner fluid, showed pronounced wrinkling of its surface as the slump went on. We attributed this to the development of a more viscous surface layer as water evaporated, and its subsequent buckling under lateral compressive stresses. The feature suggests that over longer times, the flow experiences additional resistance, which lowers

2 The actual material used was not pure kaolin, but diluted joint compound - a commercially available kaolin-based building material. The joint compound has the advantage of separating slowly, but the disadvantage of containing other chemicals that initiate changes in its properties over longer timescales. 


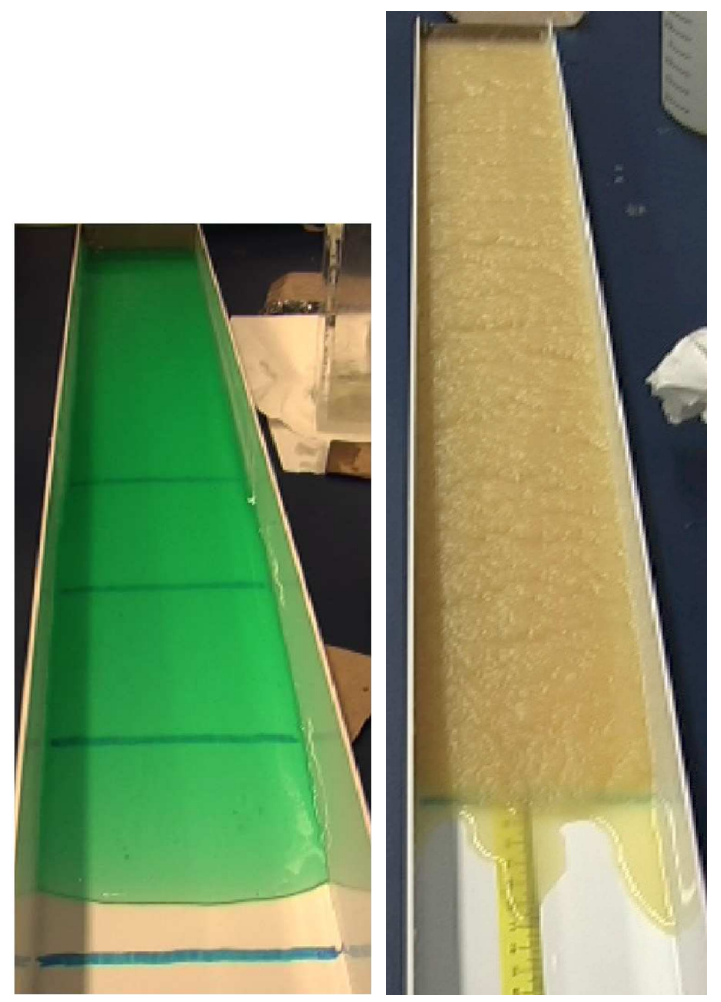

Fig. 6. Photograph of (a) dyed carbopol and (b) apple sauce slumping down the channel. The flow front is relatively flat, suggesting that the slump is largely two-dimensional and the side walls do not have much effect. The channel is $10 \mathrm{~cm}$ wide and $6 \mathrm{~cm}$ deep (well over $1 \mathrm{~m}$ long), and the initial reservoir was 40 cm long. The lines drawn across the channel in (a) indicate the original position of the dam and the three stations downstream of it where recordings of the passing fluid front were made. In (b), note the fluid at the flow front that has separated from the suspension, and the uneven surface of the deposit.

the time ratios, as observed. Nevertheless, it seems feasible to use the time ratios as diagnostics for cornsyrup.

A more detailed comparison of the experiment with numerical solutions of the (Newtonian) thin-layer model is shown in figure 9. To reconstruct dimensional time, the theory requires a value for the viscosity; a good fit to the data is achieved by taking $\eta=4 \mathrm{~Pa}$ secs, which is consistent with (though slightly lower than) measurements in a cone-and-plate rheometer. (We made no attempt to match perfectly the temperature of the material in the rheometer and in the channel, which is a significant source of error for corn syrup owing to the strong temperature dependence of its viscosity). The comparison illustrates fairly quantitative agreement, hence we conclude that the thin-layer model provides an accurate description of this experiment.

The success of reproducing the flow behaviour in the $10 \mathrm{~cm}$-wide channel, prompted us to proceed a little further and compare the theory with slumps in a channel with half that width. Figure 7(a) also includes the observed front positions from two such dam breaks, and measurements of the intervals, $t_{j}$, are tabulated in table (i). A striking feature of these results is that the time ratios from the narrow channel closely match those from the wide channel (see the table, and also figure 8 , in which the data is included). This is not because the side walls are having no effect, as can be seen by comparing the $t_{j}$-intervals themselves, or the evolution in figure 7 (a). In fact, the slump in the narrower channel is significantly braked by the sidewalls and proceeds about 40 percent slower (as also shown by table (i), the same trend holds for apple puree). 

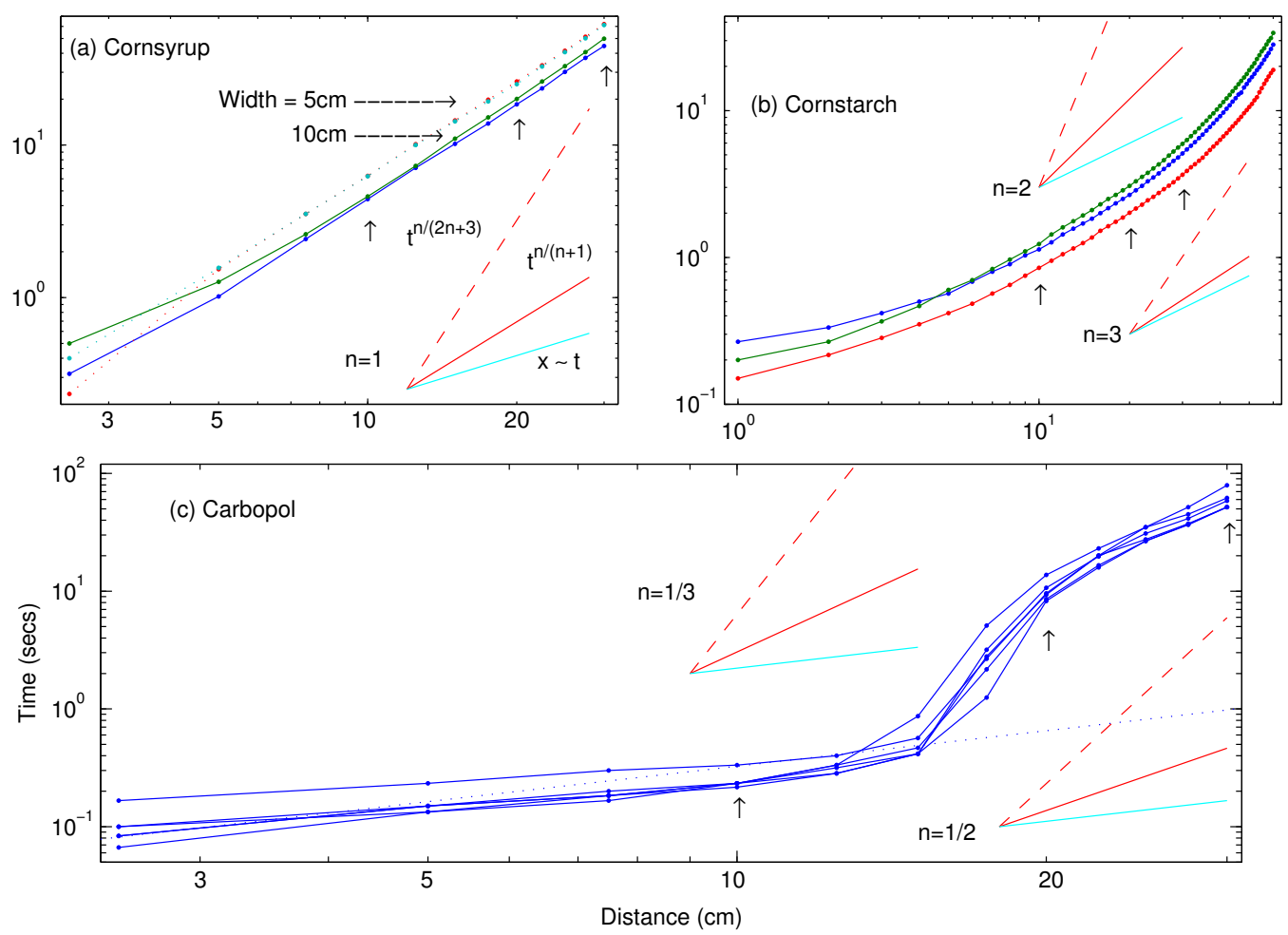

Fig. 7. Front position (measured in $\mathrm{cm}$ from the gate) against time (in seconds) for (a) cornsyrup $\left(\rho \approx 1.4 \mathrm{~g} / \mathrm{cm}^{3}\right)$, (b) cornstarch $\left(\rho \approx 1.25 \mathrm{~g} / \mathrm{cm}^{3}\right)$, and (c) carbopol (with a density just over $1 \mathrm{~g} / \mathrm{cm}^{3}$ ). The lines show the expected similarity solutions for fluid without yield stress (short-time, $x \sim t^{n /(n+1)}$; long-time, $x \sim t^{n /(2 n+3)}$; inviscid, $x \sim t$ ). The arrows indicate when the fluid passes the stations at $x=50$, 60 and $70 \mathrm{~cm}$. For the cornsyrup in panel (a), results from two channels of different width are shown (the standard one with a width of $10 \mathrm{~cm}$, and a second with half that width). In panel (c), the inviscid similarity scaling, $x \sim t \sqrt{g H}$ is also shown by the dotted line.

Despite this, the front position still progresses according to the similarity solution, which is why the time ratios come out as before. The retardation of the dam break by the side walls therefore largely acts to increase the effective viscosity, which is filtered out in taking the time ratios. We conclude that side-wall friction likely has significant effect in the Bostwick consistometer, which translates to an error in the inferred viscosity, but that the time-ratios diagnostic for rheology may be relatively insensitive.

\subsection{Xanthan gum}

Xanthan gum solutions (about 1 percent by weight) also show fair agreement with theory: The measured time ratios in figure 8 suggest that this material is a shear-thinning, power-law fluid with an exponent, $n$, close to $1 / 4$. Measurements of viscosity in a cone-and-plate rheometer do indeed suggest that 


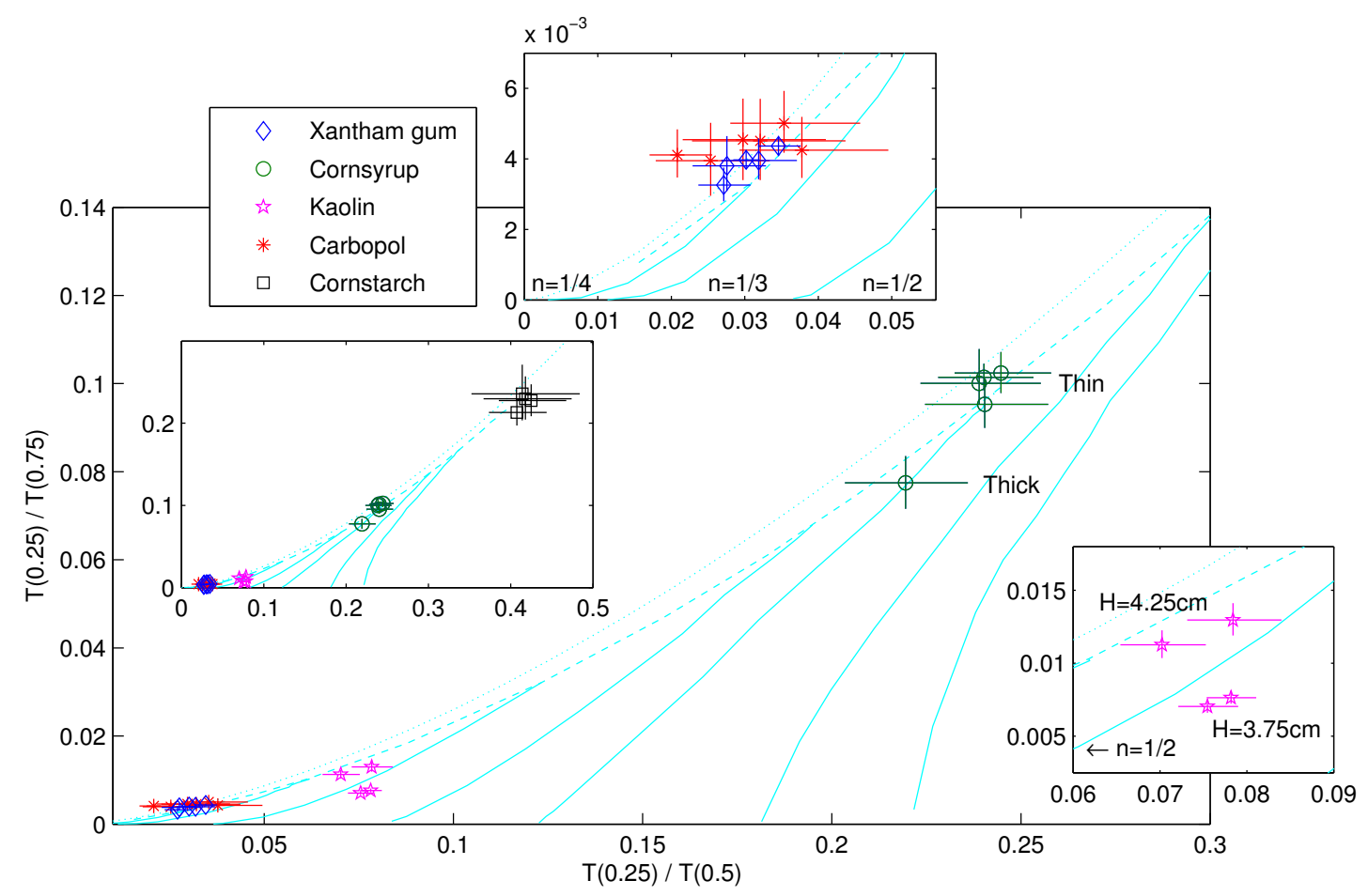

Fig. 8. Measured time ratios for corn syrup $(H=2 \mathrm{~cm})$, xanthan gum $(H=2.2$ to $2.5 \mathrm{~cm})$, kaolin $(H=3.75$ and $4.25 \mathrm{~cm})$, carbopol $(H=6 \mathrm{~cm})$ and cornstarch $(H=2 \mathrm{~cm})$. These are superposed on the curves of figure 5 .

this material can be fitted with a constitutive law of this kind (with $n \approx 0.27$ giving a slightly better fit).

Given the exponent $n$, we may further use the time measurements, $t_{j}$, and their dimensionless counterparts, $T_{j}$, to infer the consistency:

$$
K_{j} \equiv \frac{H}{L} \rho g\left(\frac{H t_{j}}{L T_{j}}\right)^{n},
$$

for $j=1,2$ and 3. For a good fit, all three should equal each other and any value measured in a rheometer. For the slump with initial depth $2.2 \mathrm{~cm}$, the observations predict $\left(K_{1}, K_{2}, K_{3}\right)=(5.73,6.11,5.98)$ m.k.s., which are consistent with one another, but greater than a value determined in a coneand-plate rheometer (which was about 4 m.k.s.). This discrepancy could arise from various sources of error in the dam break experiments (such as inertia, as we outline presently). But it is also conceivable that the value from the coneand-plate rheometer fails to agree because of intrinsic problems with wall slip (which such devices are prone to - see Barnes [7])

A full comparison of theory and experiment for the evolution of the front position is shown in the second panel of figure 9. The main panel shows the comparison adopting the inferred value of 6 m.k.s.; the inset shows the poorer 

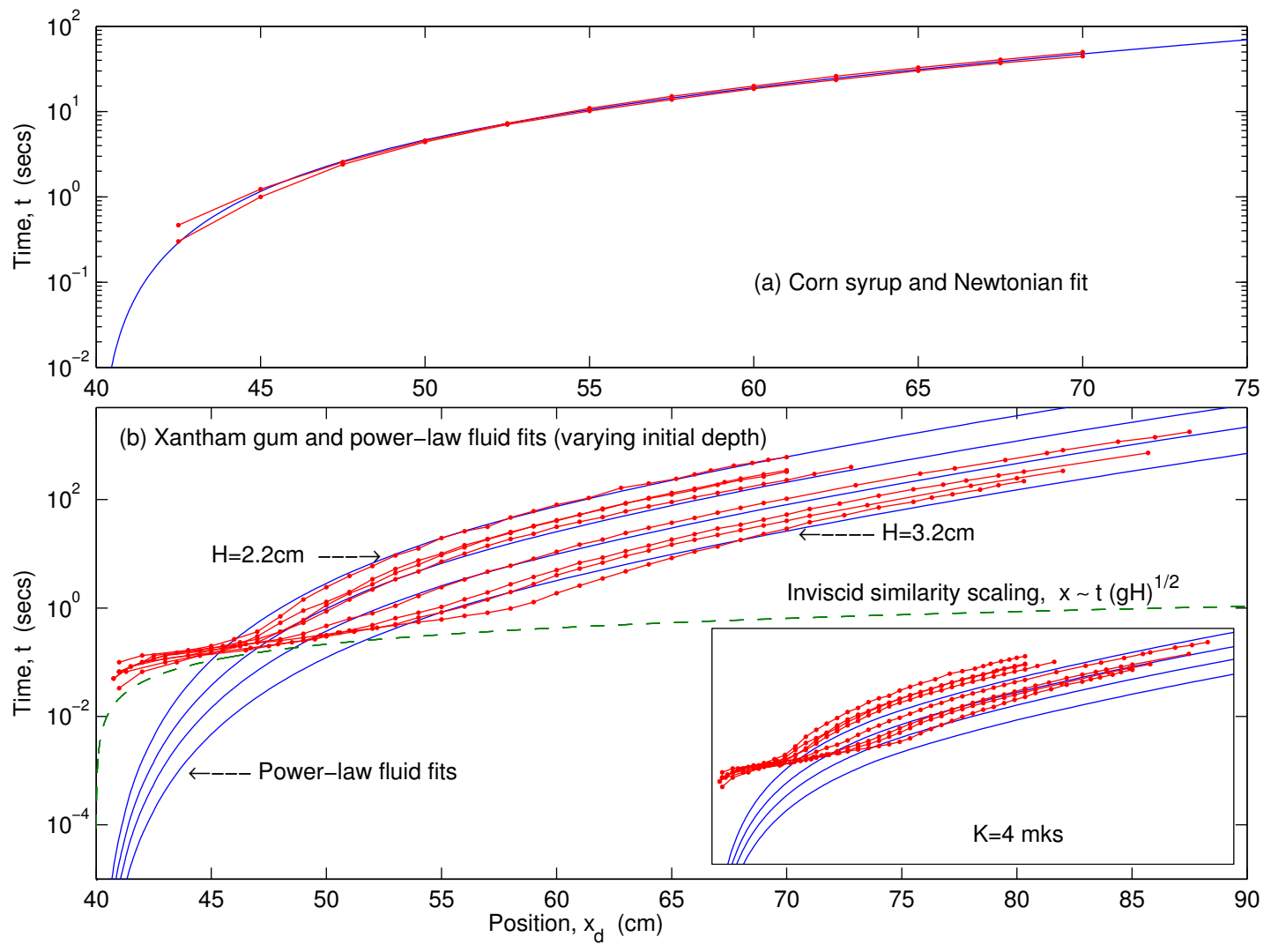

Fig. 9. Front positions (measured from the back wall; dots and lines) against time for (a) (lower viscosity) cornsyrup $(H=2 \mathrm{~cm})$ and (b) xanthan gum (for four different initial depths, $H=2.2,2.5,2.8$ and $3.2 \mathrm{~cm}$ ). The curves show the theoretical results expected based on the viscosity of the thinner cornsyrup and the xanthan gum rheology inferred from the recorded times, $t_{1}, t_{2}$ and $t_{3}$ (which give $4 \mathrm{~Pa}$ secs as the viscosity of the cornsyrup and $n \approx 0.27$ and $K \approx 6$ m.k.s. units for the gum). In the second panel, the inviscid similarity scaling is also displayed (with $H=2.2 \mathrm{~cm}$ ); the inset shows the theoretical curves if $K=4 \mathrm{~m} . \mathrm{k} . \mathrm{s}$. is used for the consistency, as was measured in a cone-and-plate rheometer (and with the same power-law exponent).

comparison if the rheometric value is used instead. Although the comparison is by and large fairly satisfactory, there is some significant disagreement at early times. For this fluid, the dam breaks are relatively fast at the outset, and it is likely that inertia plays a prominent role in the early stages of the slump. Indeed, the front positions shown in figure 9 follow a path more like that expected from inviscid similarity scaling. As one raises the initial depth, inertial effects become yet more important, so much so that they significantly increase the time ratios. Data showing the dependence of the time ratios on $H$ is displayed in figure 10. Thus, inertial effects limit the diagnostic potential of these measurements. 
Fluid Width $(\mathrm{cm}) \quad t_{1}$ (secs) $\quad t_{2}$ (secs) $\quad t_{3}$ (secs) $\quad t_{1} / t_{2} \quad t_{1} / t_{3}$

$\begin{array}{lllllll}\text { Corn syrup } & 10 & 4.43 & 18.5 & 45.4 & 0.24 & 0.098 \\ & 5 & 6.32 & 26.1 & 67.0 & 0.24 & 0.102\end{array}$

$\begin{array}{lllllll}\text { Apple sauce } & 10 & 0.48 & 13.3 & 90.2 & 0.036 & 0.0052 \\ & 5 & 0.95 & 24.6 & 163.2 & 0.036 & 0.0055\end{array}$

Table 1

A comparison of times and time ratios for corn syrup and apple sauce (density of about 1.4 and $1.04 \mathrm{~g} / \mathrm{cm}^{3}$, respectively) in channels of $10 \mathrm{~cm}$ and $5 \mathrm{~cm}$ width. The measurements quoted are averages over all the experimental runs undertaken. For the apple sauce, the narrower slumps began with slightly higher initial depths than in the wider channel (about $4 \mathrm{~cm}$, as compared to $3.6 \mathrm{~cm}$ ) to avoid excessive fluid separation.

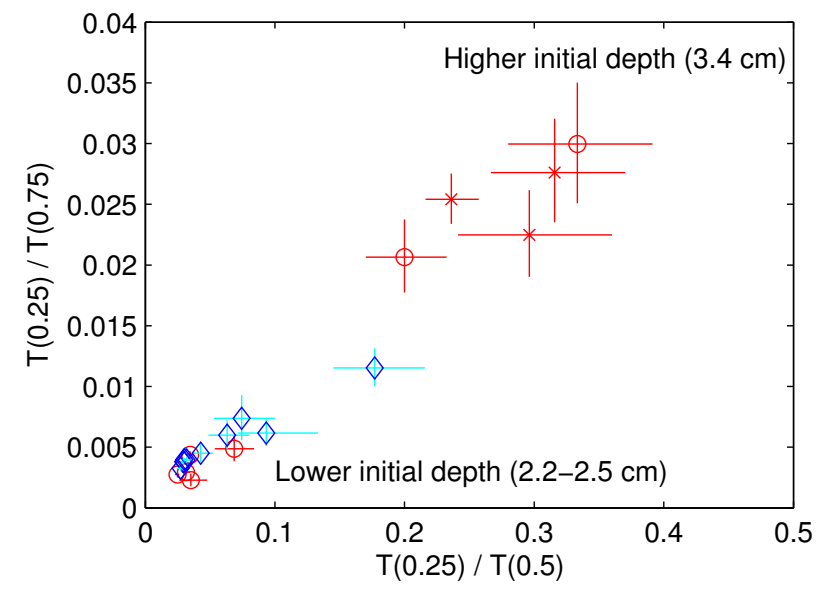

Fig. 10. Measured time ratios for xanthan gum for varying initial depth, $H$, ranging from $2.2 \mathrm{~cm}$ upto $3.4 \mathrm{~cm}$.

\subsection{Kaolin}

The time ratios for kaolin (joint compound) suggest a shear thinning material with an exponent, $n \approx 0.5$. In addition, the data are now significantly below the curve expected for power-law fluid, confirming the presence of a yield stress. The experiments shown in figure 8 are performed with two initial 
heights, which translates to different values for $B$. One expects that the lower initial reservoirs have larger Bingham numbers and therefore time ratios, a trend that is roughly seen in the data. A comparison of the shallower data with the theoretical time ratios suggest a rheological fit of $B$ in the range $0.1-$ 0.15. Unfortunately, although we were able to confirm the detailed power-law dependence of the viscosity in the cone-and-plate rheometer (which indicated $n \approx 0.6$ ), we were unable to measure a reliable yield stress, presumably due to significant wall slip.

The kaolin slumps also eventually decelerate towards a yield-stress dominated final state. We show evidence for this in figure 11, which displays the advance of the fluid edge in the four experiments: Towards the end of the slumps, the front position veers upwards to approach its final constant value. Because the slump comes to rest relatively slowly (with expected dependence $t^{-1}$ ), it is difficult to observe the approach to the final state in detail (especially because other factors come into play on long timescales, such as evaporation from the surface layers and surface tension). The inset shows a measured final profile for the shallower slump, together with a theoretical fit for $B=0.18$ (which suggests that the deeper slumps are characterized by $B=0.13$ ). Adopting $n=0.6$, we compute corresponding numerical solutions, and with the observed values of $t_{j}$, we further estimate that $K \approx 30 \pm 10$ m.k.s. units, using (17). The theoretical, dimensional results that then follow are also shown in the main panel of figure 11. There is some disagreement in the initial stages of the slump (again probably due to inertia), but the later stages compare fairly well, if not fully satisfactorily (and the rheological fit has not been optimized).

\subsection{Carbopol}

Turning next to carbopol (0.1\% carbopol 940 in water, neutralised with Sodium Hydroxide), we see that the time ratios (figure 8) are close to what might expected for a shear-thinning fluid with a low yield stress. A closer examination, however, uncovers some significant discrepancies: First, cone-and-plate rheometry suggests a yield stress of order a few $\mathrm{Pa}$ and a viscosity exponent of around $0.4-0.5$ for the fluid used. For the initial fluid depth and length (about $6 \mathrm{~cm}$ and $40 \mathrm{~cm}$, respectively), we then estimate Bingham numbers of order 0.05 . But given those estimates of $n$ and $B$, we observe from figure 8 that the measured ratios are actually quite different from those expected theoretically; the carbopol data ought to be closer to that for kaolin.

Second, the advance of front shown in the final panel of figure 7 also disagrees with theoretical predictions. In particular, at early times the flow is closer to the inviscid similarity solution, indicating that inertia plays a major role in the initial phases of the slump. On the other hand, the later times do seem to 


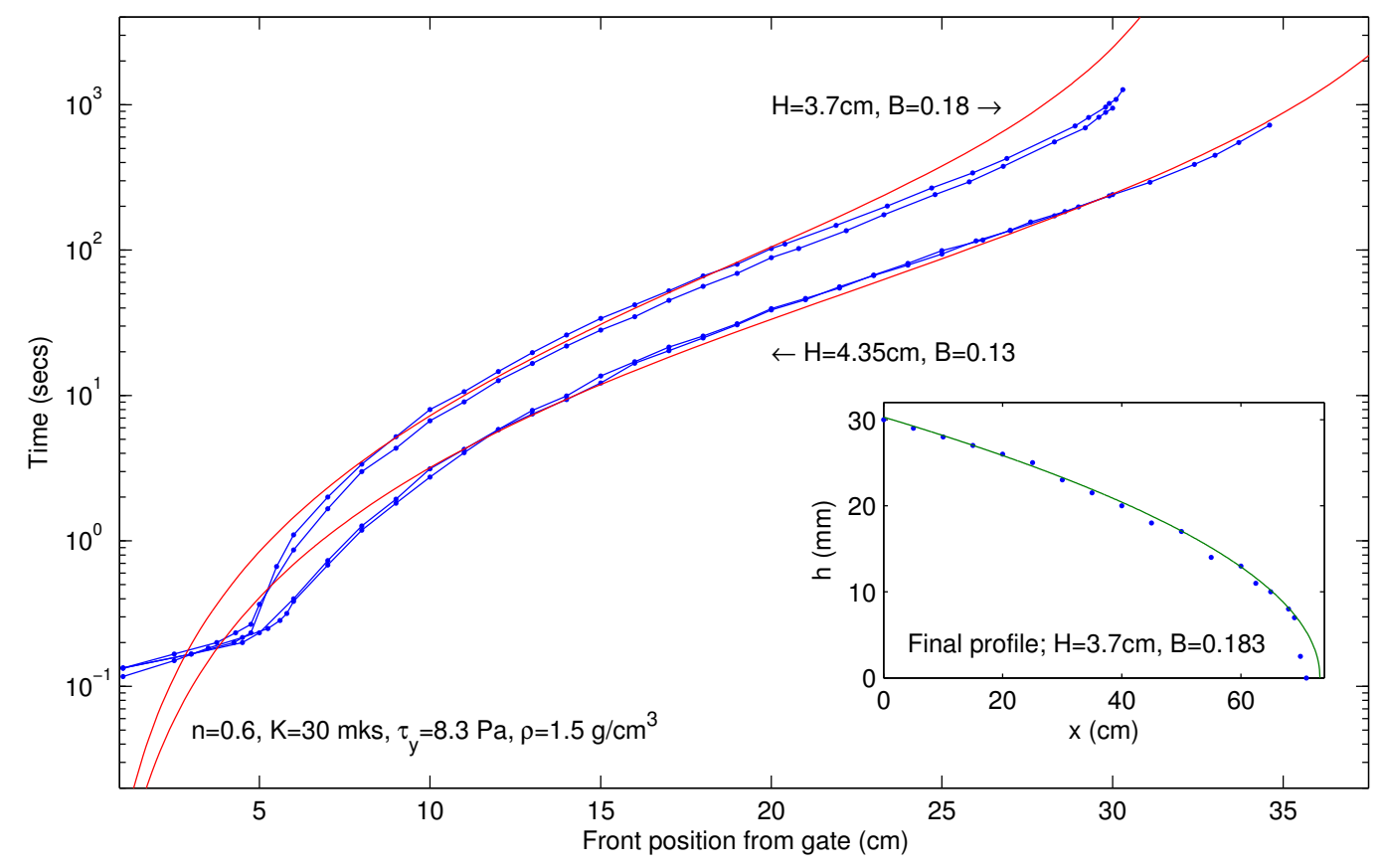

Fig. 11. Evolution of the fluid edge (dots and lines), as measured from the gate, for kaolin suspensions (density $1.5 \mathrm{~g} / \mathrm{cm}^{3}$ ). The curves show theoretical solutions given the Herschel-Bulkley fit indicated. The inset compares the final profile of one of the shallower slumps (dots) with the expected equilibrium state (curve).

be fit quite well by the viscous similarity solution if the power-law exponent, $n$, is about $1 / 2$. The trouble is brought out clearly by figure $7(\mathrm{c})$ : the first time interval, $t_{1}$, lies in the inertial regime, and the second, $t_{2}$, lies close to a transitional phase; only the third time instant lies in the power-law regime. Thus, the time ratios partly reflect inertial effects.

Part of the reason why inertia was relatively strong for the carbopol dam breaks was that the initial depth was fairly large $(6 \mathrm{~cm})$. To try to reduce inertial effects, we repeated some of the dam breaks with a shallower initial fluid layer ( $H$ in the range 3.2 to $3.8 \mathrm{~cm}$ ). These flows had ratios that were indeed closer to those expected, based on the estimated values of $n$ and $B$. However, they were also well above the power-law curve in figure 8 (lying close to $(0.05,0.015)$ for $H=3.8 \mathrm{~cm}$, and $(0.08,0.023)$ for $H=3.2 \mathrm{~cm})$, indicating that problems still remained (possibly wall slip).

Overall, then, the time ratios appears less informative about the carbopol rheology than they are for cornsyrup and xanthan gum. On the other hand, from a qualitative perspective, the ratios do successfully distinguish the fluid from a Newtonian one and suggest that carbopol is shear thinning. Significantly, tracing the front position appears useful in pinpointing when inertia is overly important. 


\subsection{Cornstarch}

Time ratios for cornstarch (with concentration 52 percent by weight, and density of about $1.25 \mathrm{~g} / \mathrm{cm}^{3}$ ) lie to the right of and above the viscous time ratios in figure 8, as expected for a shear thickening material. Indeed, the ratios suggest that this material is a power-law fluid with an exponent of $n=3$ or 4 . There is slender evidence from cone-and-plate rheometry (performed by S. Mandre \& A. C. Rust, personal communication) that this material's steady state shear rheology can be fit by such a model, and is certainly shear thickening. Tracking the front position (figure $7(\mathrm{~b})$ ), on the other hand, indicates that the evolution is not well fit by a power law. In fact, some flows of higher concentration look to stop after travelling about a metre whilst remaining about $1 \mathrm{~cm}$ deep at the back of the channel. This suggests the presence of a small yield stress of half a $\mathrm{Pa}$ or so. The material also clearly fractures and bends like a solid when the gate is raised, and the initial dam break does not look at all like those of the

other fluids used. Suffice to say that this is a curious material that warrants further study.

\section{$5 \quad$ Purees and Bostwick tests}

\subsection{Apple sauce}

We close our experimental exploration by mentioning some dam breaks performed with apple sauce as an example of a real fruit puree. The fibrous, coarse structure of this material and its tendency to separate (i.e. watery fluid to collect at the fluid edges; see figure 6(b)) precludes a serious investigation in standard rheometers. The latter also prevents us undertaking overly long dam breaks.

Sample experiments are shown in figure 12. The time ratios observed suggest a power-law fluid with exponent close to $n=1 / 4$. A more detailed fit of the front evolution shows fair agreement, except for an inertial initial transient. These stages of the slump show little sign of a yield stress in the puree. However, over a longer period, the fluid appears to break towards a halt; a "final profile" is displayed in the figure and suggests a yield stress of around $3 \mathrm{~Pa}$. This translates to a Bingham number of around $B=0.09$, which is larger than expected based on the time ratios, but not completely inconsistent with them. Furthermore, experiments with more concentrated apple puree showed smaller values for the time ratio, $t_{1} / t_{3}$, as expected if the yield stress were larger.

As for cornsyrup, when we break the dam in a narrower channel, even though 


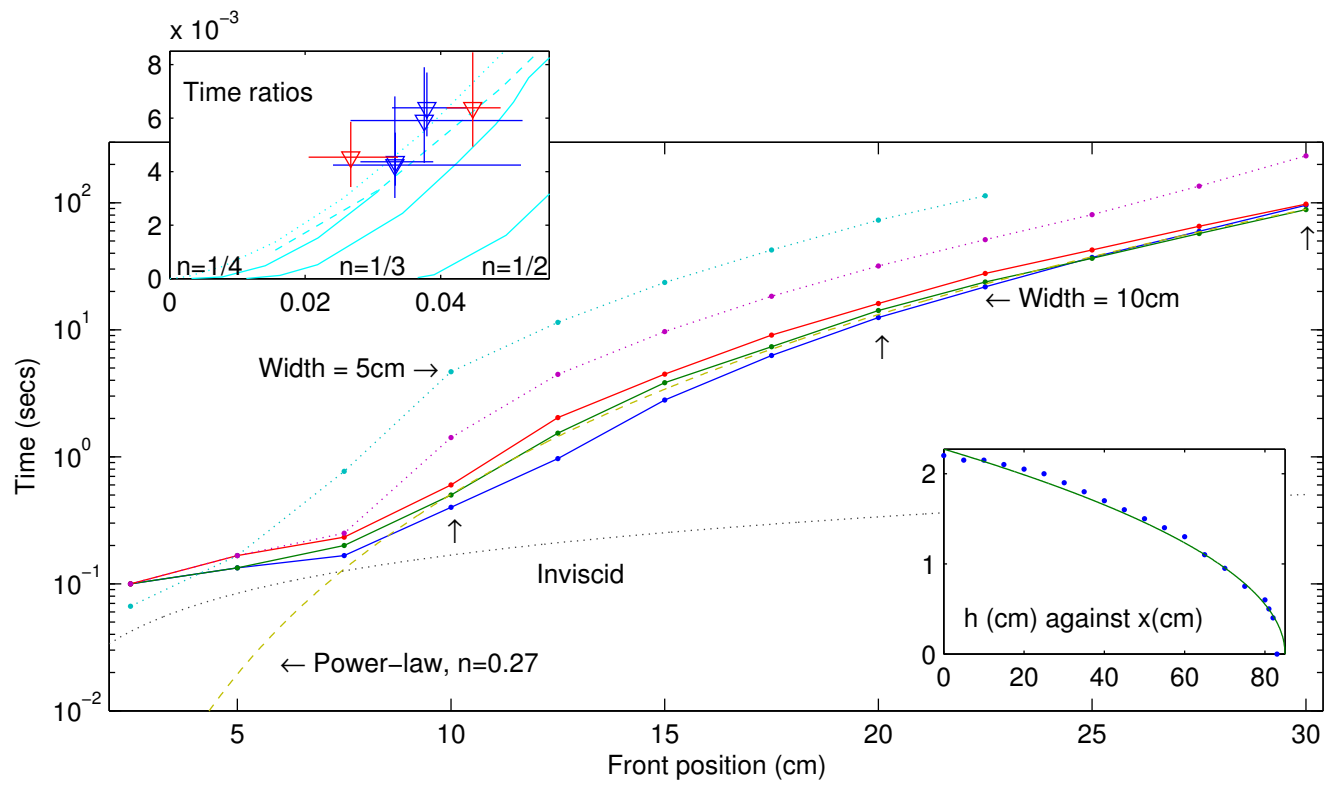

Fig. 12. Dam breaks with apple sauce ("Sun-Rype" brand, with about 25 percent extra water; $\left.H=3.6 \mathrm{~cm}, \rho \approx 1.04 \mathrm{~g} / \mathrm{cm}^{3}\right)$. The main panel displays the evolution of the fluid edge (measured from the gate) for dam breaks in the usual (10 cm wide) and narrow ( $5 \mathrm{~cm}$ wide) channels. For the narrower channel, the initial depth was increased to $4 \mathrm{~cm}$ to avoid excessive fluid separation at the nose of the slump over the duration of the experiment. The insets show the time ratios and the profile of a final shape (together with a theoretical fit using $B=0.093$ ). The time ratios for the wider channel are shown by the darker (blue) points; the lighter (red) points indicate the ratios for the narrower channel.

the observed time ratios do not change very much, the overall progression of the slump is significantly slowed (see also the values included in table (i)). If one were to measure the distance travelled after a given time period (as in the $B_{30}$ measurement), one would therefore consistently overestimate the internal strength of the material.

Some words of caution are in order here, though: as the fluid slumps down the channel, separation clearly occurs at the fluid's leading edge, making the identification of this position ambiguous and the final deposit inhomogeneous. Indeed, the surface of the deposit is relatively rough and shows evidence of fairly regularly spaced, sharp steps that might be due to fracture or rupture of the fibrous microstructure (see figure 6(b)).

Worse still, the flow of the apple sauce was substantially reduced when the floor of the channel was roughened by covering it with coarse (50 grit, garnet) sandpaper. Roughening a smooth wall is a common trick in rheometry to try to reduce effective wall slip. Most commonly, such slip arises because of the development of a relatively dilute fluid layer adjacent to the wall that lubricates the remainder of the material. When we tried this trick with the channel, it became immediately clear that the flow was significantly affected 

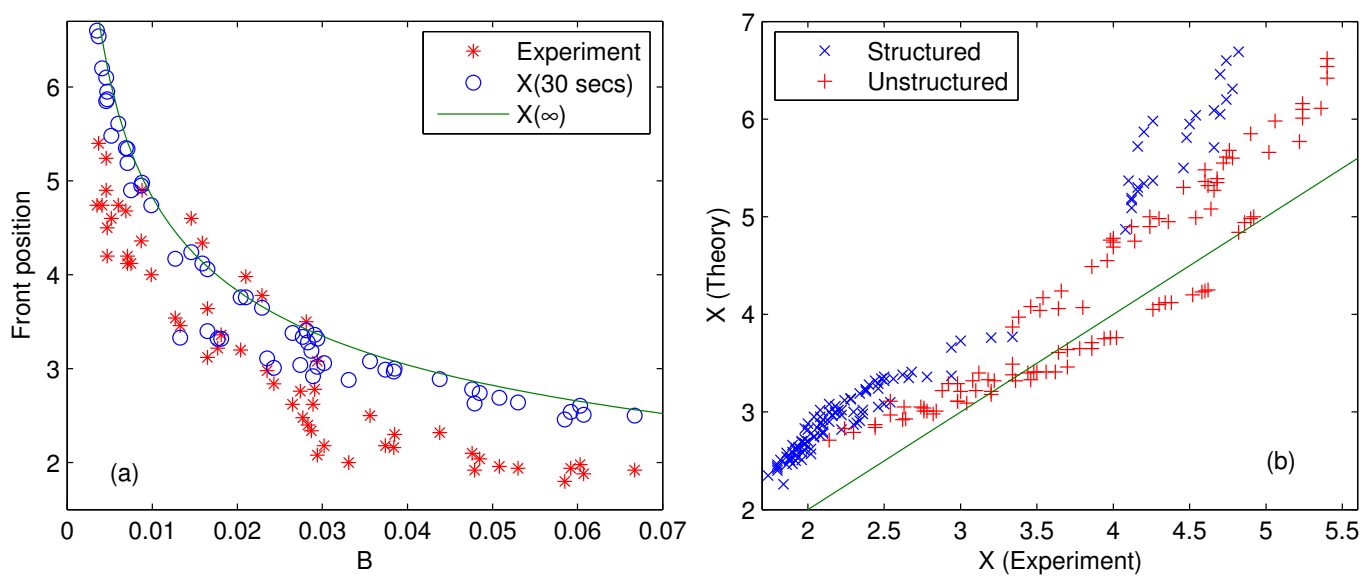

Fig. 13. Experimental and theoretical front positions for fruit purees in the Bostwick rheometer. Panel (a), shows experimental front positions after 30 seconds (stars), together with the corresponding theoretical positions (circles) after an equivalent time (and using the rheological data tabulated by Perona [17]). The curve shows the expected final state. Panel (b) plots the experimental position against the theoretical one. Data after 5, 10, 30 and 60 seconds are included; the crosses indicate "structured" purees, whilst the plusses denote "unstructured" ones.

by wall slip: a fluid layer that virtually came to rest on the sand paper after flowing only ten or so centimeters, could easily propagate on the smooth channel much faster and over twice the distance. Given the large amount of separation evident at the flow front, this pronounced effect of slip is perhaps not so surprising. However, it does suggest that in devices like the Bostwick rheometer there is likely significant wall slip as a result of separation, which must cloud any rheological inference (see also the discussion in [17]).

\subsection{Comparison with the Bostwick tests}

Figure 13 shows a compilation of data from the experiments reported by Perona [17]. The first panel of this figure shows front positions after 30 seconds against $B$, determined using Perona's tabulated rheological measurements. This data is compared with numerical solutions of the thin-layer model, with the circles indicating the theoretical position after a dimensionless time equivalent to the 30 seconds, and the curve showing the final, stationary position. The difference between the circles and line offers an estimate of how much the slump is still moving. Evidently, although some of the slumps are close to their final state, others are not. This suggests that the $B_{30}$ measurement cannot be safely used to infer yield stress in all situations.

Figure 13(a) also clearly shows that the experimental front positions are systematically below their theoretical counterparts. The degree of discrepancy is brought out further in the second panel of the figure, which plots theoreti- 


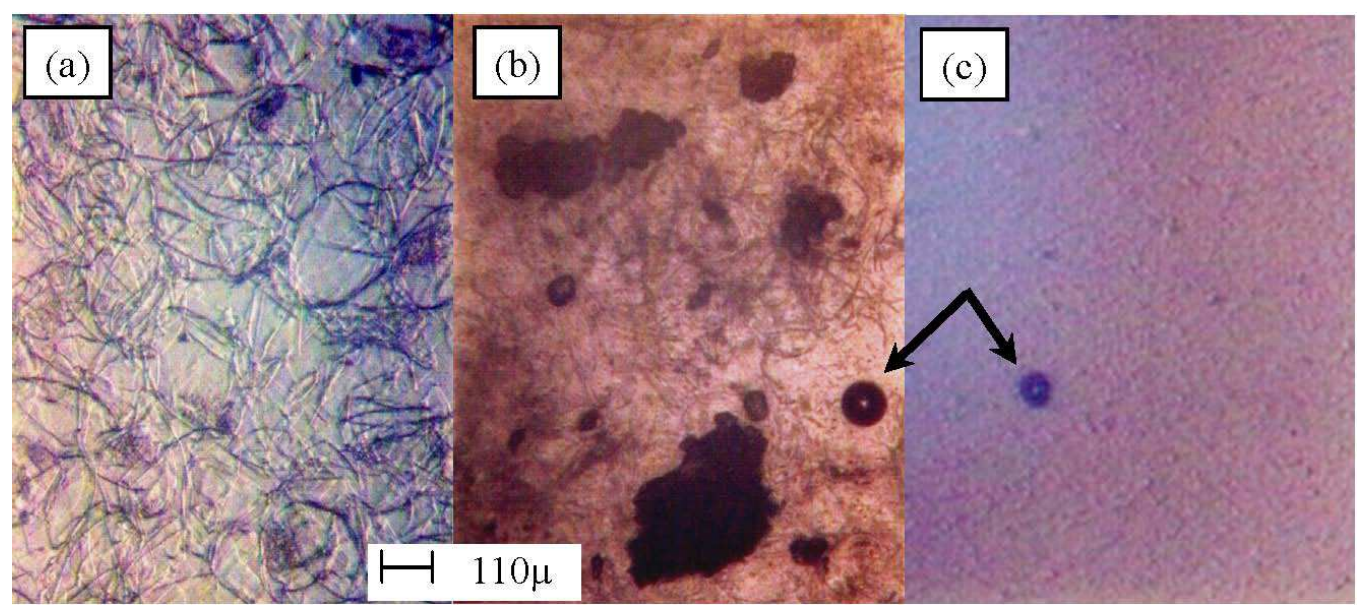

Fig. 14. Microscopic images of examples of "structured" and "unstructured" purees: apple (panel (a)) and pear (panel (b)) purees show typical hair-like and irregular solid fibres, respectively; lemon treacle (panel (c)) is much smoother in comparison. Arrows indicate some trapped air bubbles (spherical dark spots). Whilst apple puree could still show a marked coiled structure at high dilution rates, this effect was less pronounced for pear.

cal against experimental front position, and includes positions measured at times of 5, 10, 30 and 60 seconds. Part of the discrepancy could be due to inertia limiting the initial accelerations, as for the xanthan gum experiments. However, some of the slumps are not particularly shallow, and the Bostwick consistometer is also relatively narrow $(5 \mathrm{~cm})$. Hence, sidewall resistance is correspondingly more important, and our earlier comparison between channels of width $10 \mathrm{~cm}$ and $5 \mathrm{~cm}$ (figures 7 and 12; table (i)) indicates that this could easily explain the observed errors. More curiously, the data also appear to divide quite cleanly into "structured" and "unstructured" fluids. That is, into measurements for purees that possess a very fibrous, entangled microstructure, and purees that do not (we illustrate the two types of purees in figure 14). The data for structured purees compares least favourably with the theory, suggesting that there could also be some systematic rheological bias in the Bostwick tests (other possible errors in the rheological fits are described by Perona [17]). Nevetheless, to explore the possibility in more detail, and give a more complete comparison between theory and experiment, one requires an extension of the thin-layer model that incorporates both inertia, side-wall resistance and (perhaps most importantly) wall slip.

\section{Discussion}

In this article we have explored a shallow-fluid theory intended to model the dambreak and slump of a viscoplastic fluid in a relatively wide channel. We complemented the theory with an experimental study in which we released 
various fluids in a laboratory channel, including corn syrup and aqueous suspensions of xanthan gum, kaolin, carbopol and cornstarch. The problem also has some connection with the so-called "Bostwick consistometer", used in food science, and we have drawn some brief comparisons between the two. We have explored in part whether a series of three measurements (the times taken for the flow front to reach three fixed stations down the channel) can be used to gain a more complete picture of the rheology than the usual Bostwick measurement (the distance travelled over a fixed time period).

For the experimental arrangement that we used (a channel of $10 \mathrm{~cm}$ width and with fluid depths of between 2 and $6 \mathrm{~cm}$ ), the thin-layer theory appears to model adequately the dam break of corn syrup and xanthan gum, aside from an initial period of adjustment wherein inertial effects play a role. The diagnostic time ratios predict that these fluids are Newtonian and shear-thinning, respectively, to a degree that compares well with measurements in a cone-andplate rheometer. Although there is some disagreement between the inferred yield stress and that measured in the rheometer, the time-ratio diagnostics for the kaolin suspensions also predict that this fluid is shear thinning and possesses a yield stress. Unfortunately, the same cannot be said for carbopol, which appears to be overly influenced by inertia and other effects; the time ratios suggest only that this fluid is strongly shear thinning. Cornstarch suspensions are predicted to be shear thickening, as expected, but little further insight is given into this curious material.

The partial success of our time-ratio diagnostic suggests that the basic idea may well be worth pursuing in the future. First, we have made no attempt to optimize the choice of the stations at which the time intervals are measured. It may prove worthwhile to consider other positions in order to try to "straighten out" the wedge-shaped region of the time ratios so that it becomes more rectangular and easier to discriminate nonlinear viscous behaviour from the yield stress. We have also performed a series of fairly crude experiments to compare with theory. Better techniques for the release of the dam and the monitoring of the advance of the front would certainly reduce the errors. We also made only a cursory attempt to compare systematically the rheological inferences with direct measurements from rheometers (partly because of wall-slip problems in our cone-and-plate device), and determine the influence of the channel dimensions (varying the initial fluid depth and channel width a little, and the reservoir length not at all). Lastly, as we have already mentioned there is scope to improve the theory by adding inertia, side-wall friction and effective slip over the channel floor. Turned around, perhaps one could use the dambreak as an experimental device to understand and parameterize these effects (especially wall slip). However, it is also not clear that the Herschel-Bulkley fit is adequate of itself; thixotropy, for example, is known to be important in the slumps of some viscoplastic materials $[10,11]$. 
Acknowledgements: This work was initiated at the BIRS Workshop on Viscoplastic fluid mechanics, October 2005. We thank BIRS for support. We thank Mark Martinez and Andy Hogg for discussions.

\section{A The stopping problem}

The ultimate convergence to the final state can be analytically addressed with perturbation theory [15]. We summarise the relevant results here and place them into our current notation; we put $n=1$ and describe only the Bingham case. We first transform into a moving coordinate frame, $(x, t) \rightarrow(\Xi, t)$, determined by the instantaneous positions of the upstream and downstream flow fronts, $x_{u}(t)$ and $x_{d}(t) \equiv X(t)$ respectively:

$$
\Xi=\frac{x-x_{u}(t)}{x_{d}(t)-x_{u}(t)}
$$

thus, $\Xi=0$ refers to the back edge and $\Xi=1$ to the advancing front. The upstream edge, $x=x_{u}(t)$, always lies inside the fluid layer for $B>1 / 3$, but if $B<1 / 3$, there is an instant when it reaches the back wall and thereafter remains there.

The transformation can be introduced into the governing equation, which, as the solution converges towards the final state, simplifies to

$$
h_{t} \longrightarrow-\frac{1}{2} B\left(Y^{2}\right)_{x}
$$

giving

$$
h_{t}-\frac{\dot{x}_{u}+\Xi\left(\dot{x}_{d}-\dot{x}_{u}\right)}{x_{d}-x_{u}} h_{\Xi}=-\frac{B\left(Y^{2}\right)_{\Xi}}{2\left(x_{d}-x_{u}\right)}
$$

in the new coordinate system, where

$$
Y \longrightarrow h+\frac{B}{h_{\Xi}}\left(x_{d}-x_{u}\right) .
$$

Next, one adopts a separable solution of the form,

$$
h(\Xi, t)=H(\xi)+\eta(\Xi) \Lambda(t)
$$


where $\xi$ is the infinite-time limit of the moving coordinate, $\Xi$. Taking $|\Xi-\xi|$ and $|\eta|$ to be small, and exploiting separability, the governing equation reduces to the two relations,

$$
\dot{\Lambda}=-\kappa B^{2} \Lambda^{2}
$$

and

$$
\kappa\left(\eta+\delta \xi H_{\xi}-X_{u} H_{\xi}\right)=\frac{\partial_{\xi}\left[(H \eta)_{\xi} H_{\xi}+\delta H\right]^{2}}{2 B\left[x_{d}(\infty)-x_{u}(\infty)\right]},
$$

where

$$
\delta=\frac{x_{d}-x_{u}}{\Lambda\left[x_{d}(\infty)-x_{u}(\infty)\right]}-\frac{1}{\Lambda}, \quad X_{u}=\frac{x_{u}(t)-x_{u}(\infty)}{\Lambda\left[x_{d}(\infty)-x_{u}(\infty)\right]}
$$

and $\kappa$ is the separation constant. The second equation is more transparently written in terms of the variables,

$$
\tau=\sqrt{1-\xi} \equiv \frac{H(\xi)}{H(0)} \quad \text { and } \quad Q=\frac{H}{A}\left(\eta+\frac{1}{2} \delta H\right)
$$

which give

$$
\frac{k}{2}\left(Q_{\tau}^{2}\right)_{\tau}=1-Q
$$

where

$$
A=\frac{B}{\Lambda}\left[x_{d}(\infty)-x_{d}(t)\right] \quad \text { and } \quad \kappa=\frac{A}{k[H(0)]^{3}}
$$

Equation (A.6) implies the relatively slow rate of convergence, $t^{-1}$ :

$$
\Lambda=\frac{1}{\Lambda^{-1}(0)+\kappa B^{2} t}
$$

This rate can be seen immediately, if more qualitatively, from (A.3) because the time derivative, $h_{t}$, is quadratic in the perturbation amplitude (i.e. $Y$ ). The other relation (A.8) is solved subject to the boundary conditions that we choose the regular solution, $Q \sim \tau^{3 / 2}$, at the singular points where $Q_{\tau}=0$ (which coincide with $\tau=Q=0$ and $\tau=1=Q / 2$ ). This simultaneously determines the separation constant, $k=3 / 4 \times[\beta(2 / 3,2 / 3)]^{-3} \approx 0.0866$ (with $\beta(r, s)$ denoting the Beta function). 

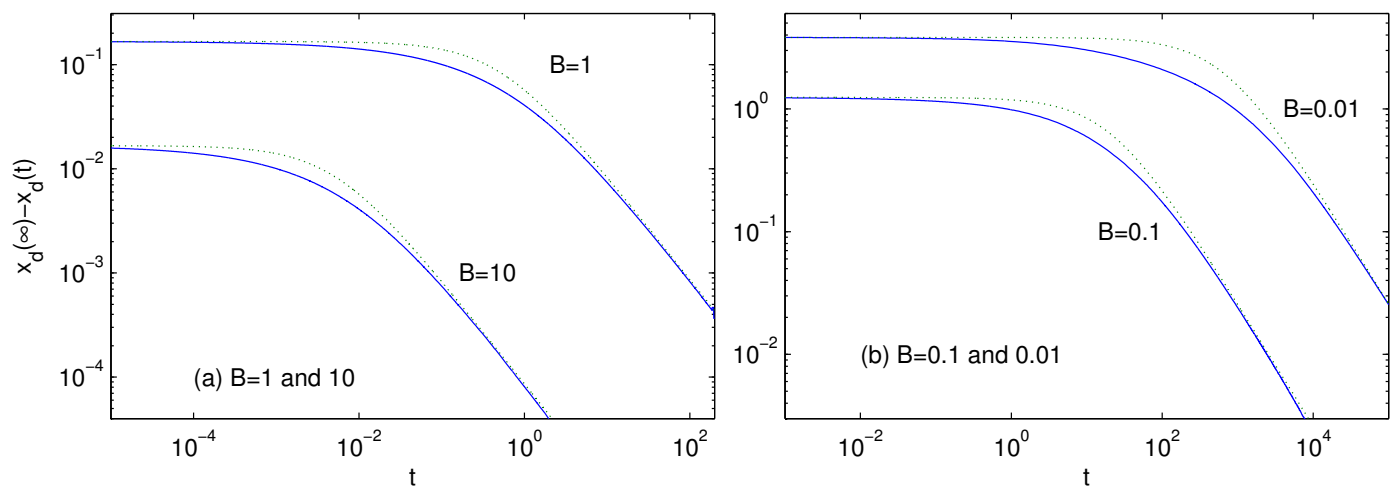

Fig. A.1. Convergence of the downstream front to its final position for (a) $B=1$ and 10, and (b) $B=0.1$ and 0.01. $n=1$. In each case, the expected theoretical fit is shown by the dotted curves.

We collect together the results for the two cases, $B>1 / 3$ and $B<1 / 3$ separately: For $B>1 / 3$, the boundary conditions at $\xi=1$ further imply that $x_{u}(t)-x_{u}(\infty) \equiv x_{d}(\infty)-x_{d}(t)$. Moreover, $H(0)=1$. Consequently,

$$
x_{d} \sim 1+\frac{1}{6 B}-\frac{1}{6 B}\left(1+\frac{B^{2} t}{6 k}\right)^{-1},
$$

if we make the assumption that $x_{d}(0)=1$ (which is of course true, but a little unrealistic in the perturbation theory). This prediction is compared with numerical computations in figure A.1. Conversely, if $B<1 / 3$, then $x_{u}=X_{u}=$ 0 and $H(0)=\sqrt{3 B}$, giving

$$
x_{d} \sim\left(\frac{9}{8 B}\right)^{1 / 3}-\left[\left(\frac{9}{8 B}\right)^{1 / 3}-1\right]\left\{1+\frac{B^{2} t}{3 k}\left[\left(\frac{9}{8 B}\right)^{1 / 3}-1\right]\right\}^{-1} .
$$

Again, this is compared to numerics in figure A.1.

Lastly, we may compute the expected stopping times, $T_{\text {stop }}$, assuming that this is adequately predicted by the long-time asymptotics: Taking $x_{d}=1+$ $0.99\left[x_{d}(\infty)-1\right]$ implies that

$$
T_{\text {stop }} \approx\left\{\begin{array}{ll}
51 / B^{2}, & B>1 / 3 \\
\left(26 / B^{2}\right) /\left[(9 / 8 B)^{1 / 3}-1\right], & B<1 / 3
\end{array} .\right.
$$

These predictions compare very nicely with the data obtained numerically, as shown in figure 3. A glance at the evolution sequences in figure A.1 indicates why this should be so: by the time the front has moved to within 1 percent of its final value, the solution is well into the asymptotic regime. 


\section{B Numerical details}

To cope with the discontinuities at $x_{d}(t)$ and $x_{u}(t)$, it is convenient to change coordinates, $(x, t) \rightarrow(\Xi, t)$, as defined by equation (A.1). (The failure to deal with the discontinuities leads to small numerical oscillations near those points which gradually poison the numerics.) The governing equation is then

$$
\begin{aligned}
h_{t}= & \frac{1}{\left(x_{d}-x_{u}\right)^{1+1 / n}} \frac{\partial}{\partial \Xi}\left[\frac{n\left|h_{\Xi}\right|^{1 / n-1} Y^{1+1 / n}}{(n+1)(2 n+1)}[(1+2 n) h-n Y] h_{\Xi}\right] \\
& +\frac{\dot{x}_{d} \Xi h_{\Xi}}{\left(x_{d}-x_{u}\right)}+\frac{\dot{x}_{u}(1-\Xi) h_{\Xi}}{\left(x_{d}-x_{u}\right)}
\end{aligned}
$$

where $Y=h-\left[B\left(x_{d}-x_{u}\right)\right] /\left|h_{\Xi}\right|$ and $0 \leq \Xi \leq 1$. Spatial discretization is performed using a simple piecewise nonlinear Galerkin method, along the lines suggested by Skeel \& Berzins [19]: We construct a grid indexed by $1 \leq i \leq N$, where $N$ the total number of gridpoints. Let

$$
\hat{\Xi}_{i+\frac{1}{2}}=\frac{\Xi_{i+1}+\Xi_{i}}{2}, \quad h\left(\hat{\Xi}_{i+\frac{1}{2}}\right)=\frac{h_{i+1}+h_{i}}{2}, \quad h_{\Xi}\left(\hat{\Xi}_{i+\frac{1}{2}}\right)=\frac{h_{i+1}-h_{i}}{\Xi_{i+1}-\Xi_{i}} .
$$

Then,

$$
h_{t}(i)=2 \frac{g_{i+\frac{1}{2}}-g_{i-\frac{1}{2}}}{\Xi_{i+1}-\Xi_{i-1}}+\frac{\Xi_{i+1}-\Xi_{i}}{\Xi_{i+1}-\Xi_{i-1}} r_{i+\frac{1}{2}}+\frac{\Xi_{i}-\Xi_{i-1}}{\Xi_{i+1}-\Xi_{i-1}} r_{i-\frac{1}{2}} .
$$

where $g\left(i+\frac{1}{2}\right)$ and $r\left(i+\frac{1}{2}\right)$ are the flux and rescaling terms in (B.1) computed at $\hat{\Xi}_{i+\frac{1}{2}}$. At the left boundary we impose the no-flux condition,

$$
h_{t}(i=1)=\frac{g_{\frac{3}{2}}}{\hat{\Xi}_{\frac{3}{2}}}+r_{\frac{3}{2}} .
$$

Provided $x_{u}>0$, ordinary differential equations for the front positions are obtained by setting $h_{t}(i=1)=0$ in (B.4) and $h_{t}(i=N)=0$ in (B.3). Those equations are then coupled to the discretized $h$-equation and the whole system is evolved in time using a fifth-order backward differentiation formula (Gear's method). When $B<1 / 3$, the slumping material reaches the back wall at a certain time which is precisely located and the integration stopped. It is then resumed, but with $x_{u}$ fixed at zero; we still set $h_{t}(i=N)=0$ in (B.3) to obtain an evolution equation for $\dot{x}_{d}$, but equation (B.4) now provides an equation for $h_{t}(i=1)$. 
To account carefully for the steep front at $x_{d}$, the points along the $\Xi$-axis are non-uniformly spaced and are collocated in $[0,1]$ according to the relation $d \Xi_{i} / d i=\varsigma(i-1)^{N_{0}}(i-N)^{N_{1}}$ where $\varsigma$ is set so that $\Xi_{N}=1$. The parameters $N_{0}$ and $N_{1}$ increase the densities of points at the extremes. We typically employed $N_{0}=0, N_{1}=2$ and $N=400$. Finally, volume conservation is not explicitly enforced as part of the computational scheme; numerical checks upon the solutions show that it is conserved.

\section{References}

[1] Balmforth, N. J., Bush, J. W. M. \& R. V. Craster, R. V. 2005 Roll waves on flowing cornstarch suspensions. Phys. Letts. A 338, 479-484.

[2] Balmforth, N. J. \& Craster, R. V. 1999 A consistent thin-layer theory for Bingham fluids. J. Non-Newtonian Fluid Mech. 84, 65-81.

[3] Balmforth, N. J., Craster, R. V. \& Sassi, R. 2003 Shallow viscoplastic flow over an inclined plane. J. Fluid Mech. 470, 1-30.

[4] Balmforth, N. J., Craster, R. V. \& Sassi, R., 2004 Dynamics of cooling viscoplastic domes. J. Fluid Mech. 499, 149-182.

[5] Balmforth, N. J., Craster, R. V., Rust, A. C. \& Sassi, R., 2006 Viscoplastic flow over an inclined surface. J. non-Newtonian Fluid Mech., this issue.

[6] Balmforth, N. J., Ghadge, S. \& Myers, T., 2006 Surface-tension-driven fingering in a viscoplastic fluid. J. non-Newtonian Fluid Mech., this issue.

[7] BARnes, H. A. 1995 A review of the slip (wall depletion) of polymer solutions, emulsions and particle suspensions in viscometers: Its cause, character, and cure. J. Non-Newtonian Fluid Mech. 56, 221-251.

[8] BARnes, H. A. 1999 The yield stress - a review or ' $\pi \alpha \nu \tau \alpha \rho \epsilon \iota$ ' - everything flows? J. Non-Newtonian Fluid Mech. 81, 133-178.

[9] Blake, S. 1990 Viscoplastic models of lava domes. In Lava flows and domes: emplacement mechanisms and hazard implications (ed. J. H. Fink), pp. 88-128. IAVCEI Proc. in Volcanology, vol. 2, Springer-Verlag.

[10] Coussot, P., Nguyen, Q. D., Huynh, H.T. \& D. Bonn. 2002 Avalanche behaviour in yield-stress fluids. Phys. Rev. Lett 88, 175501.

[11] Coussot, P., Roussel, N., Jamy, S. \& H. Chanson. 2005 Continuous or catastrophic solid-liquid transition in jammed systems.

[12] Huppert, H. 1982 The propagation of two-dimensional and axisymmetric viscous gravity currents over a rigid horizontal surface J. Fluid Mech. 121, $43-58$. 
[13] LiU, K. F. \& MEI, C. C. 1989 Slow spreading of Bingham fluid on an inclined plane. J. Fluid Mech. 207, 505-529.

[14] Lyman, A. W., Kerr, R. C, \& Griffiths, R. W. 2005 Effects of internal rheology and surface cooling on the emplacement of lava flows. J. Geophys. Res. 110, B08207.

[15] Matson, G. P. \& A. J. HogG. 2006 J. Non-Newtonian Fluid Mech., this issue.

[16] McKarthy, K. L. \& J. D. Seymour. 1994 Gravity current analysis of the Bostwick consistometer for power law foods. J. Texture Stud. 25, 207220

[17] Perona, P. 2005 Bostwick degree and rheological properties: An up-to-date viewpoint. Appl. Rheol. 15, 218-229.

[18] Piau, J. M. \& K. Debiane. 2005 Consistometers rheometry of power-law viscous fluids J. Non-Newt. Fluid Mech, 127, 213-224.

[19] Skeel, R. D. \& M. Berzins. 1990 A method for the spatial discretization of parabolic equations in one space variable. SIAM J. Sci. Stat. Comput. 11, 132. 